\title{
Die Entwicklung des österreichischen Abstammungsrechts in der NS-Zeit im Lichte der Rechtsprechung des LGZ/LG Wien und des Reichsgerichts
}

\begin{abstract}
The transformation of Austrian lineage law in the light of the jurisdiction of the Regional Civil Court in Vienna and the 'Reichsgericht'

As part of the research project "Privatrecht in unsicheren Zeiten - Österreichische Zivilrechtsjudikatur unter der NS-Herrschaft" (Private Law in Perilous Times - Austrian Civil Law Judicature under National Socialist Rule), the author was given the opportunity to write a doctoral thesis in which the development of Austrian lineage law during the period of National Socialism was thoroughly reviewed for the first time. This article presents a summary of the research and its results: Judgements of the Vienna Regional Court and the Austrian Senate of the 'Reichsgericht' are used to analyse how the judges dealt with the contradiction between statute and ideology. In addition to the legal framework, other factors that were decisive for the courts, such as the introduction of new types of evidence and the influence of National Socialist and state authorities, are also considered.
\end{abstract}

Keywords: Austria - Civil law - Law of lineage - Marriage law - National Socialism - Paternity

\section{Rechtspolitische Forderungen und gesetzliche Änderungen in der NS-Zeit}

\section{a. Die Umdeutung und -wertung des Abstammungsrechts}

Das Abstammungsrecht war eines jener Rechtsgebiete innerhalb des Zivilrechts, deren Regelungen in einem Spannungsverhältnis zu mehreren fundamentalen weltanschaulichen Elementen des Nationalsozialismus standen. Den NS-Juristen ging es dabei vor allem um die Harmonisierung von rechtlichen Termini aus dem Familienrecht mit den NS-Konzepten zu „Erbgesundheit" und „Rasse“. So wurde eine stärkere Berücksichtigung der „materiellen Wahrheit" im Bereich der rechtlichen Eltern- schaft und eine stärkere Betonung biologischer Aspekte bei der rechtlichen Definition von Abstammung, Familie, Vater- und Mutterschaft gegenüber sozialen Anknüpfungspunkten gefordert. Hinzuweisen ist jedoch darauf, dass die "rassische Einteilung" der Bevölkerung in „Volkszugehörige“ und „Blutsfremde“, die sich selbst als „biologisch“ verstand, ${ }^{1}$ in ihrer zentralen definitorischen Grundlage - den Nürnberger Gesetzen - auf der Religionszugehörigkeit der Großeltern basierte. Dennoch wurde gleichzeitig besonderer Wert darauf gelegt, durch Förderung "rassebiologischer Forschung“ dem ideo-

\footnotetext{
${ }^{1} \mathrm{Zu}$ den prä- und protonationalsozialistischen Rassenkonzepten und ihren Grundlagen im akademischen Diskurs der Zeit siehe u.a. MAYER, Familie, Rasse und Genetik 162.
} 
logischen Konzept wissenschaftliche bzw. akademische Legitimation zu verleihen.

Der sich schrittweise verschärfende Prozess der Diskriminierung bestimmter sozialer Gruppen, die sich in erster Linie auf Juden i.S.d. NSRassekonzepts bezog, ging mit der Einführung von Verfahren einher, die es ermöglichten, Personen den jeweiligen Gruppen rechtlich zuzuordnen.

Die Bedeutung der Gruppenzugehörigkeit beschränkte sich anfänglich auf die Arbeit im Staatsdienst, von der in Deutschland ab 1933 Personen "nicht arischer Abstammung“ ausgeschlossen wurden. ${ }^{2}$ In weiterer Folge zielte die NS-Politik auf eine umfassende "rassische“ Einteilung der Staatsbevölkerung ab, die mit Diskriminierungen in allen Lebensbereichen einherging. Entsprechende Nachweise der "rassischen Einordnung“, die ab 1936 als „Abstammungsbescheide" bezeichnet wurden, stellte die Dienststelle des Sachverständigen für Rasseforschung aus. Der Name dieser Dienststelle wurde später auf Reichsstelle für Sippenforschung bzw. 1940 auf Reichssippenamt geändert (in Folge: Reichssippenamt). In dem Verfahren vor dem Reichssippenamt wurde anhand der Dokumente die Abstammung überprüft. Nur in Zweifelsfällen wurden rassebiologische Gutachten eingeholt, die der Entscheidung eine scheinbar wissenschaftliche Legitimation geben sollten.

Da diese Gutachten verwaltungsrechtliche Entscheidungen darstellten, unterlag deren Anfechtung ex lege dem Verwaltungsverfahrensrecht. ${ }^{3}$ Entsprechend finden sich unter den im Zuge des

$2 \S 3$ Gesetz zur Wiederherstellung des Berufsbeamtentums (RGBl. I 1933, 175); Einführung in Österreich durch RGB1. I 1938, 1225. Gemäß § 2 (1) 1. DVO des Gesetzes zur Wiederherstellung des Berufsbeamtentums waren die tatbestandsmäßigen Voraussetzungen für „nicht arische Abstammung“ bereits mit dem Vorhandensein eines nicht arischen, insbesondere jüdischen Großelternteils erfüllt.

${ }^{3}$ RdErl des RMI IV 501 8b 15.8 vom 26. 10. 1934, vgl. WALK, Sonderrecht für die Juden 95.
Projekts untersuchten Urteilen des Landgerichts für Zivilrechtssachen bzw. Landgerichts Wien (in Folge: LGZ/LG Wien) ${ }^{4}$ auch Zurückweisungen von Klagen, die inhaltlich auf eine „Feststellung der rassischen Abstammung" abzielten und für die sich das LGZ/LG Wien daher nicht zuständig sah. ${ }^{5}$

In Deutschland lässt sich ab Mitte der 1930er Jahre ein wachsender indirekter Einfluss der im deutschen Zivilrecht vorgesehenen Abstammungsanfechtungen ${ }^{6}$ auf die "rassische Einordnung" konstatieren. Denn in der Regel legten die zuständigen Verwaltungsbehörden ihren Abstammungsnachweisen Eintragungen in den Geburtsmatriken zugrunde und eine Anfechtung dieser Eintragungen war gesetzlich vor den Zivilgerichten vorgesehen.7 So gesehen bestand die Möglichkeit, über das Zivilverfahren konkrete Änderungen der Matriken zu bewirken und somit indirekt den Inhalt eines Abstammungsbescheides $\mathrm{zu}$ beeinflussen. Dadurch, dass von dieser Möglichkeit in der Praxis vermehrt Gebrauch gemacht wurde, erwuchs dem Abstammungsverfahren eine Bedeutung, die der ursprüngliche Gesetzgeber freilich nicht vorhergesehen hatte. Auch der nationalsozialistische Gesetzgeber reagierte auf diese Entwicklung nur zögerlich.

4 Ab 1.5. 1939 wurden das Landgericht für Zivilrechtssachen, die Landgerichte für Strafsachen I und II, das Handelsgericht und der Jugendgerichtshof in Wien als Landgericht Wien bezeichnet (RGBl. I 1939, 751).

${ }^{5}$ Klagen auf Feststellung der rassischen Abstammung wurden von den Zivilgerichten zurückgewiesen: LG Wien 2.12.1941, 10 Cg 375/41 (unbekannt); LG Wien 4.1.1943, 1 Cg 329/42 (unbekannt).

${ }^{6} \mathrm{Zu}$ den unterschiedlichen Rechtsverhältnissen im deutschen Abstammungsrecht und den jeweiligen Anfechtungsmöglichkeiten vgl. BUKOR, Zivilrecht und Ideologie 93-105.

${ }^{7} \S 48$ des Personenstandsgesetzes vom 3.11.1937, RGBl. I 1937, 1146; Einführung des Personenstandsgesetzes in Österreich durch RGBl. I 1938, 803. 


\section{b. Die Gesetzeslage nach dem ABGB}

Nach dem ABGB gab es mehrere Anfechtungsmöglichkeiten, die sich darin unterschieden, wie das zugrundeliegende Vaterschaftsverhältnis zustande gekommen war: durch Geburt des Kindes innerhalb der Ehe, durch nachträgliche Legitimierung im Zuge einer späteren Eheschließung oder durch Anerkennung der unehelichen Vaterschaft. Von den Klagen, die vor dem LGZ/LG Wien eingebracht wurden, stellte sich in der Praxis die Anfechtung der ehelichen Vaterschaft als wichtigste heraus. ${ }^{8}$ Die Aktivlegitimation kam dabei in erster Linie Ehemännern $\mathrm{zu}$, die sich betrogen glaubten und sich daher weigerten, Unterhaltszahlungen für das potentiell außerehelich gezeugte Kind zu leisten. Nur wenn der gesetzliche Vater vor Ablauf der Anfechtungsfrist starb oder er seit Geburt des Kindes unbekannten Aufenthaltes war, konnte eine Anfechtung von Dritten (z.B. erbberechtigten Verwandten, seit der ABGB-Teilnovelle 1914 auch vom Kind selbst) eingebracht werden. Die Passivlegitimation der Ehelichkeitsanfechtungsklage kam einem Kurator zur Verteidigung der ehelichen Abstammung $\mathrm{zu}$, der im Verfahren die Interessen des Kindes wahrzunehmen hatte.

Die gesetzlichen Bedingungen für eine erfolgreiche Ehelichkeitsanfechtung waren für den Kläger sehr ungünstig. Zum einen konnte der Kläger die Klage nur innerhalb von drei Monaten ab Kenntnisnahme von der Geburt einbringen der Gesetzgeber hatte in erster Linie das Kindeswohl und nicht die Übereinstimmung der biologischen mit den rechtlichen Verhältnissen im Auge -, zum anderen lag die Beweispflicht beim Kläger und der Nachweis der NichtVaterschaft war in der Praxis schwer zu erbringen.

\footnotetext{
${ }^{8}$ Daneben existierte noch die Anfechtung von legitimierten Vaterschaften, die ebenfalls vor dem Landesgericht bzw. Landgericht erfolgte, sowie die Anfechtung der unehelichen Vaterschaft, die beim Bezirksgericht bzw. Amtsgericht einzubringen war.
}

\section{c. Die Änderungen der gesetzlichen Rahmenbedingungen}

Während im sogenannten "Altreich" - dem Staatsgebiet des Deutschen Reiches, wie es vor dem "Anschluss" Österreichs bestanden hatte schon im April 1938 eine Gesetzesänderung zu einer Anpassung an die neue Rolle des Abstammungsrechts führte, ${ }^{9}$ wurde die Anwendung des Gesetzes auf Österreich explizit ausgeschlossen. Vorerst blieben somit die abstammungsrechtlichen Bestimmungen des ABGB in Kraft. ${ }^{10}$

Dafür ausschlaggebend waren zum einen das Bestreben, ein "gesamtdeutsches Zivilrechtsbuch" zu erlassen, ${ }^{11}$ zum anderen die Unsicherheit hinsichtlich der legistischen Kompatibilität des ergangenen Gesetzes mit dem weiterhin bestehenden ABGB. Erst 1943 erfolgten mit der Verordnung über die Angleichung familienrechtlicher Vorschriften vom 6. Februar $1943^{12}$ grundlegende prozessrechtliche Änderungen, die zu einer weitgehenden Angleichung dieser Rechtsmaterie an die Rechtslage im "Altreich" führten.

Die Eingriffe in das österreichische Abstammungsrecht, die die Verordnung nach sich zog, lassen sich hinsichtlich ihrer Motive und ihrer Intensität in drei Kategorien einteilen.

Zur ersten Gruppe zählen jene Änderungen, bei denen es sich um formelle Anpassungen im Hinblick auf die Harmonisierung der Rechtsordnungen in den "Donau- und Alpengauen“ und dem "Altreich" handelte, die weder besonders ein-

\footnotetext{
${ }_{9}$ Vgl. BUKOR, Zivilrecht und Ideologie 123-126.

${ }^{10}$ Bis 1943 kam es zu einer einzigen gesetzlichen Änderung, die (auch) das Abstammungsrecht betraf. Dabei ging es um eine Hemmung von Fristen, wenn eine Partei von den besonderen Verhältnisse betroffen war, die sich aus der "gegenwärtigen politischen Lage“ ergaben. Die zeitliche Nähe des Erlasses dieser Verordnung zum Angriffskrieg gegen Polen legt den Schluss nahe, dass mit "gegenwärtiger politischer Lage“ der anstehende Krieg gemeint war.

${ }^{11}$ Zum „Volksgesetzbuch" vgl. BUKOR, Zivilrecht und Ideologie 38-44.

12 RGBl. I 1943, 80.
} 
schneidend waren noch einen ideologischen Hintergrund hatten. Dazu gehörte die Verlängerung des in § 138 ABGB (,Vermutung der Ehelichkeit") festgelegten Zeitraums von 300 Tagen auf 302 Tage nach Beendigung der Ehe, womit den Vorgaben des BGB entsprochen wurde. Die Verlängerung der Frist wurde auch damit begründet, dass die verlängerte Empfängniszeit der neueren medizinischen Forschung eher entspräche, ${ }^{13}$ wobei damit wohl versucht wurde, sachlich $\mathrm{zu}$ rechtfertigen, warum sich die Frist des BGB und nicht die des ABGB durchgesetzt hatte. ${ }^{14}$ Ein weiterer Punkt der Verordnung betraf die Stellung von Kindern, die in den ersten 180 Tagen der Ehe geboren wurden. Während für solche Kinder nach dem ABGB die Unehelichkeitsvermutung galt oder zumindest eine vereinfachte Form der Ehelichkeitsanfechtung durch den Vater möglich war, ${ }^{15}$ wurden diese nunmehr ohne jede Einschränkungen als ehelich angesehen. Geregelt wurden nunmehr auch jene Fälle (die bislang im ABGB keine Berücksichtigung gefunden hatten), in denen Kinder zwischen zwei Ehen geboren wurden, die so knapp hintereinander lagen, dass sich die Ehelichkeitsfrist der ersten Ehe mit jener der zweiten Ehe überschnitt. Hier galt das Kind fortan prinzipiell als eheliches Kind des zweiten Ehemannes.

Die zweite Gruppe von Änderungen betraf die Neugestaltung der $\S \S 156-159 b$ ABGB, die die Bedingungen modifizierte, unter denen ein bestehendes Vaterschaftsverhältnis angefochten werden konnte. Hier lässt sich ein gewisser ideologischer Einfluss des Nationalsozialismus erkennen, wobei nicht unbedingt "rassepolitische Gründe“ im Vordergrund standen. Es ging vielmehr darum, der „,blutmäßigen Wahrheit“ eine Vorrangstellung gegenüber den Kindesinteressen und dem Interesse an Ehelichkeit und Familie einzuräumen. Die

\footnotetext{
${ }^{13}$ SwOBODA, Familienrechtsverordnung 49.

${ }^{14}$ Zum "Gesetzespatriotismus" selbst unter eingefleischten "österreichischen“ Nationalsozialisten vgl. BUKOR, Zivilrecht und Ideologie 31-35.

${ }^{15}$ Dazu näher BUKOR, Zivilrecht und Ideologie 71.
}

Anfechtungsfrist wurde auf ein Jahr verlängert und begann erst ab dem Zeitpunkt zu laufen, ab dem der Ehemann Kenntnis von Umständen erlangte, die gegen die Ehelichkeit des Kindes sprachen. War der Ehemann innerhalb der letzten sechs Monate der Frist durch ein unvorhergesehenes oder unabwendbares Ereignis an der Bestreitung gehindert, wurde zudem der Lauf der Frist gehemmt, bis der Hinderungsgrund wegfiel.

Der Großteil der Änderungen lässt sich unter die dritte Gruppe subsumieren, die durch fundamentale Eingriffe in die Grundprinzipien des österreichischen Zivilverfahrens geprägt ist, mit dem primären Zweck, Interessen des NS-Staates im Zivilverfahren geltend $\mathrm{zu}$ machen. Dazu gehörte die Einführung des Staatsanwalts als aktivlegitimierte Partei in Abstammungsverfahren unter der Voraussetzung, dass der gesetzlich vermutete Vater die Frist versäumt hatte oder gestorben bzw. unbekannten Aufenthaltes war. Dieser vertrat im Verfahren die Interessen des Kindes bzw. der Nachkommenschaft, primär aber das öffentliche Interesse an der „materiellen Wahrheit", wobei sich dieses im Grunde auf Fälle, in denen die „rassische Zuordnung" auf dem Spiel stand, beschränkte. Selbst wenn der Staatsanwalt nicht als Kläger auftrat, wurden ihm Parteienrechte zuerkannt, sodass er selbständige Anträge und Rechtsmittel einbringen konnte.

Ein weiterer Punkt betraf das Prinzip der Parteienhoheit im Zivilverfahren, das in Abstammungsverfahren vor den Landgerichten aufgegeben wurde. Das zeigt sich zum einen darin, dass den Gerichten die Möglichkeit eingeräumt wurde, Tatsachen zu berücksichtigen, die nicht von den Parteien vorgebracht wurden (Prinzip der Amtswegigkeit), und dass in Abstammungsverfahren weder Versäumungs-, noch Verzichts- und Anerkenntnisurteile ergehen durften. ${ }^{16}$

Auch das Beweismittelrecht erfuhr eine Regelung, die der scheinbaren „Verwissenschaftlichung“ und

${ }^{16}$ Vgl. SWOBODA, Familienrechtsverordnung 51. 
wiederum der Förderung der „biologischen Wahrheit" dienen sollte. Während es nach österreichischem Verfahrensrecht nicht möglich war, Zwangsmittel gegen eine Partei oder einen Zeugen $\mathrm{zu}$ verhängen, wenn sich dieser weigerte, sich einer körperlichen Untersuchung zu unterziehen, ${ }^{17}$ wurde diese Möglichkeit auch für den Fall der Weigerung, sich erb- und rassekundlichen Untersuchungen zu unterziehen, explizit eingeführt.

\section{Der abstammungsrechtliche Judikaturbestand}

Der vorliegenden Untersuchung sind zwei verschiedene Bestände zugrunde gelegt, nämlich die Akten des LGZ/LG Wien im WStLA sowie die Urteile des Reichsgerichts aus der Entscheidungssammlung im Archiv des BGH in Karlsruhe. Im Folgenden werden beide Bestände näher beschrieben.

Im Rahmen des Projekts wurde zunächst der im WStLA lagernde Bestand der abstammungsrechtlichen Klagen des LGZ/LG Wien, der 2.350 Akten umfasst, ausgewertet. Das LGZ/LG Wien war nur für Ehelichkeitsanfechtungen, Anfechtungen legitimierter Vaterschaften sowie ab 1939/40 auch für „Klagen zur Feststellung der blutmäßigen Abstammung" zuständig. Beim LGZ/LG Wien wurden gelegentlich auch Anfechtungen von unehelichen Vaterschaften eingebracht, wobei diese in der Regel zurückgewiesen wurden, da gemäß $\S 49$ ZPO die Bezirksgerichte bzw. später die Amtsgerichte dafür zuständig waren.

Die zeitliche Einordnung von Akten lässt sich in der Regel schon an der Geschäftszahl erkennen, da die Zahl hinter dem Schrägstrich auf das Jahr der Klagseinbringung hinweist. Im untersuchten Bestand finden sich jedoch auch Akten, die zu unterschiedlichen Zeitpunkten zwischen 1938 und 1945 eingebracht wurden. Ursprünglich waren diese

${ }^{17} \mathrm{OGH} 2 \mathrm{Ob} 325 / 38$ EvBl. 1938/81; vgl. auch RG 17.5.1939, VIII 19/1939.
Akten nach dem Datum ihres Einlangens unter der fortlaufenden Geschäftszahl erfasst worden, 1945 erhielten dieselben Akten aber eine neue Geschäftszahl und wurden der Gerichtsabteilung 36 zugeordnet. Der Grund für diese Vorgehensweise konnte nicht eruiert werden. Im Rahmen der statistischen Auswertung wurden diese Verfahren nicht dem Jahr 1945 zugerechnet, sondern dem Jahr der tatsächlichen Einbringung.

Zur Aufarbeitung der Rechtsprechungspraxis wurde in dieser Arbeit zwischen "unauffälligen“ und „auffälligen“ Akten differenziert. Das Kriterium der Auffälligkeit war zum einen dann erfüllt, wenn aus dem Gerichtsakt hervorging, dass es sich bei der betroffenen Partei bzw. einer der betroffenen Parteien um eine Person „nicht arischer Abstammung" handelte. Zum anderen wurde ein Akt als auffällig bewertet, wenn von Seiten einer Partei oder des Gerichts für eine Neuinterpretation des Gesetzes bzw. teilweise sogar für eine Entscheidung gegen den Wortlaut des Gesetzes argumentiert wurde. Unauffällige Urteile wurden in erster Linie statistisch ausgewertet, auffällige Urteile wurden darüber hinaus einer qualitativen Analyse unterzogen.

Aus den Akten des Jahres 1938 lassen sich insgesamt 92 Abstammungsverfahren nachweisen, wobei es sich fast ausschließlich um Ehelichkeitsanfechtungen handelt. Von diesen sind sechs als auffällig im Sinne der obigen Definition zu beschreiben. Im Jahr 1939 stieg die Anzahl der Abstammungsverfahren auf 214, wobei weiterhin die überwiegende Mehrheit der Verfahren, nämlich 195 an der Zahl, Ehelichkeitsanfechtung waren. Von diesen sind wiederum sieben als "auffällig“ einzustufen.

Der Anstieg zwischen 1938 und 1939 setzte sich in dieser Weise fort und erreicht 1942 mit 542 Anfechtungen seinen Höhepunkt. 1943 lässt sich ein leichter Rückgang auf 471 Klagseinbringungen feststellen, wobei erst 1945 ein markanter Einbruch eintrat. 


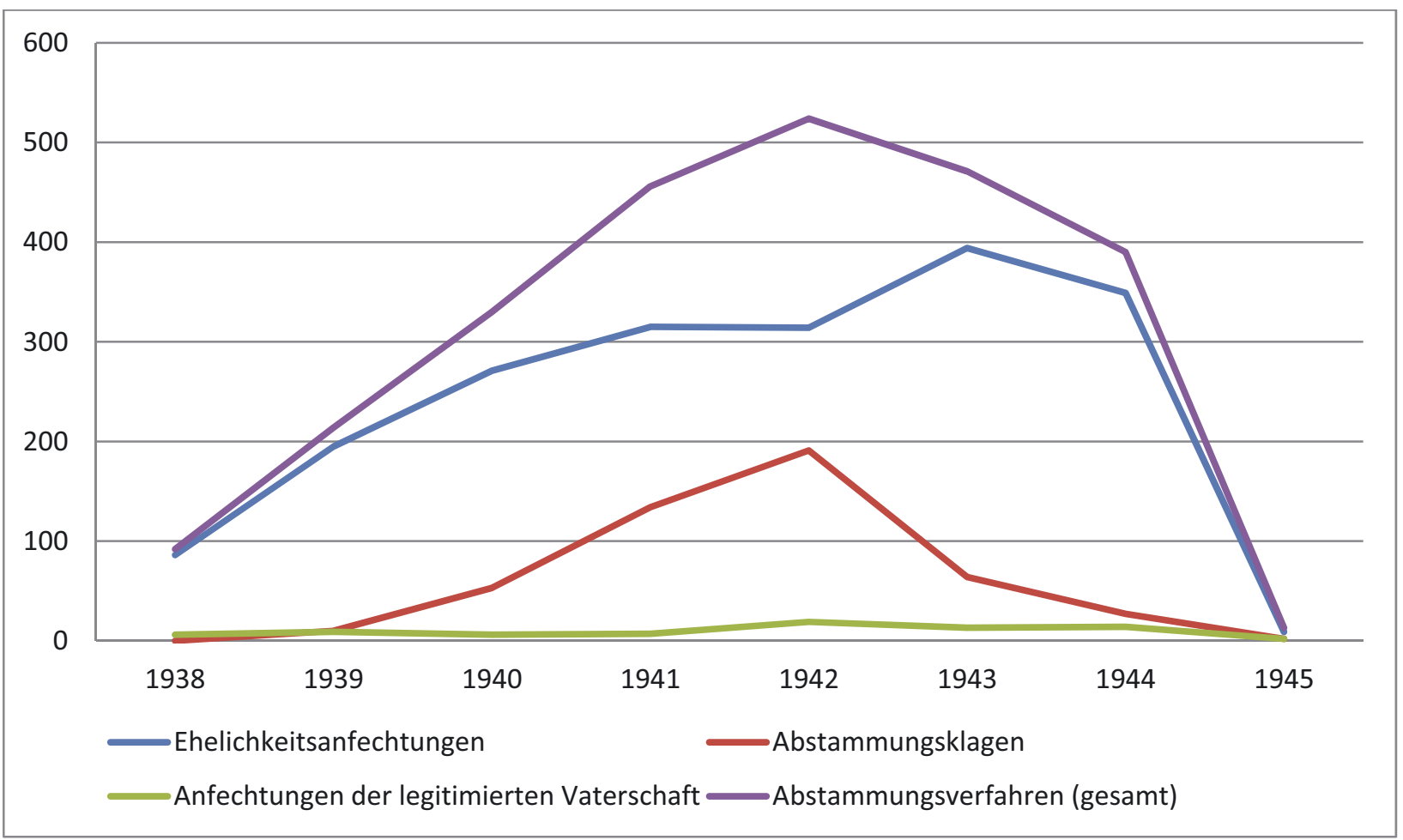

Grafik 1: Die Abstammungsverfahren vor dem LGZ/LG Wien

Darüber hinaus wurden die 99 abstammungsrechtlichen Urteile des „österreichischen Senats“ aus der Entscheidungssammlung im Archiv des BGH Karlsruhe untersucht. ${ }^{18}$

\section{Ergebnisse}

\section{a. Die Entwicklung des rechtlichen Kontextes}

Bei der Untersuchung der Rechtsprechung haben sich drei Phasen herauskristallisiert, die aufgrund der rechtlichen Rahmenbedingungen jeweils durch unterschiedliche Fragestellungen in Bezug auf die Rechtsprechung geprägt sind:

\footnotetext{
${ }^{18}$ Die in diesem Beitrag zitierten Entscheidungen des Reichsgerichts sind durchwegs der "Sammlung sämtlicher Erkenntnisse des Reichsgerichts u. Beschlüsse“ im Archiv des BGH Karlsruhe entnommen.
}

\section{Reaktionen auf den "Anschluss" (1938/39)}

Die Beibehaltung der Regeln des ABGB schuf im Lichte der geänderten Bedeutung des Abstammungsrechts Fragestellungen, die schon bald nach dem "Anschluss" auch vor dem LGZ/LG Wien landeten. Eine der zentralen Fragestellungen, die die Verfahren der Jahre 1938 und 1939 prägte, war, wie mit der Frist der $\S \S 158$, 159 ABGB umzugehen sei. Klarheit wurde hier erst mit dem Urteil des „österreichischen Senats" des Reichsgerichts vom 8. Juni 1939 geschaffen, das einer wortgetreuen Interpretation des ABGB folgte, wonach das Recht auf Anfechtung der Ehelichkeit nach Ablauf der dreimonatigen Frist ab Kenntnis von der Geburt des Kindes erlosch.

\section{Die Einführung der Abstammungsklage und die Konsolidierung der Rechtsprechung (1940-43)}

Mit der Entscheidung des Reichsgerichts, die Fristen des ABGB beizubehalten, wurde in einem nächsten Schritt die Möglichkeit geprüft, 
die vom Reichsgericht für den Geltungsbereich des BGB entwickelte Klage zur „Anfechtung der blutmäßigen Abstammung“ auch in der "Ostmark" einzuführen. Anfänglich lehnten die Richter des LGZ/LG Wien die Anwendung der Abstammungsklage durchwegs mit der Begründung ab, dass die „blutmäßige Abstammung" kein feststellbares Rechtsverhältnis sei. Erst mit der Anerkennung der Klage im Geltungsbereich des ABGB durch das Reichsgericht im Urteil vom 6. November 1939 änderte sich auch die unterinstanzliche Rechtsprechung. Die Abstammungsklage ermöglichte in Fällen, in denen die gesetzlichen Fristen abgelaufen waren, eine Anfechtung der Ehelichkeit. Einbringen konnte eine solche Klage das Kind bzw. sein gesetzlicher Vertreter, aber auch der gesetzliche Vater und die Nachkommen des „Kindes“, sofern sie ein eigenes rechtliches Interesse nachweisen konnten. In analoger Anwendung der Ehelichkeitsanfechtung des ABGB war die Klage gegen den Kurator zur Verteidigung der ehelichen Geburt einzubringen.

\section{Anpassung an die FamRechtsVO 1943 (1943-45)}

Die Rolle der Abstammungsklage erfuhr eine drastische Änderung durch die Einführung der Verordnung über die Angleichung familienrechtlicher Vorschriften vom 6. Februar 1943. Indem nach Ablauf der Frist nunmehr der Staatsanwalt die Ehelichkeit ohne zeitliche Befristung anfechten konnte, wurde der mögliche Anwendungsbereich der Abstammungsklage auf uneheliche Vaterschaften beschränkt. Auf diese Möglichkeit wurde sogar explizit in $\S 6$ der Verordnung Bezug genommen. Abstammungsklagen ehelicher Kinder waren mit Einführung der Verordnung nicht mehr möglich und wurden von den Gerichten durchwegs mangels Klagsberechtigung abgewiesen.

\section{b. Statistische Auffälligkeiten zu einzelnen Richtern und die NS-Personalpolitik ${ }^{19}$}

Im Rahmen der statistischen Analyse der Akten des LGZ/LG Wien konnte ein indirekter Zusammenhang zwischen der richterlichen Entscheidungspraxis hinsichtlich ideologisch bedeutsamer Abstammungsklagen und der späteren geschäftsmäßigen Fallzuordnung an die einzelnen Richter nachgewiesen werden. Jenen Richtern, die bei ideologisch relevanten Abstammungsklagen zu ablehnenden Entscheidungen tendierten, wurden solche ideologisch heiklen Fälle verstärkt zugewiesen. Somit wurden insbesondere Verfahren, in denen die Anfechtung der ,jüdischen Abstammung" zur Disposition stand, über Personalmaßnahmen beeinflusst. Durch einen Vergleich der vorliegenden Entscheidungen konnte eruiert werden, welche Richter wie oft für Entscheidungen herangezogen wurden und wie sich diese Zuteilung über die Jahre veränderte:

In den Jahren 1938 bis 1940 lassen sich keine Auffälligkeiten in Bezug auf die richterlichen Verfahrenszuteilung bei jenen Fällen feststellen, bei denen es um die Anfechtung der ,jüdischen" Abstammung eines Klägers ging. Im Jahre 1938 wurden insgesamt zwölf Entscheidungen zehn verschiedenen Richtern zugeteilt, ${ }^{20}$ im Jahr 193921 Entscheidungen 14 Richtern $^{21}$ und 194072 Entscheidungen 21 Richtern, $^{22}$ wobei keinem Richter

\footnotetext{
${ }^{19}$ Die letzten Absätze in diesem Abschnitt entsprechen BUKOR, Zivilrecht und Ideologie 223.

${ }^{20}$ Bei den Richtern handelt es sich um: Brachmann, Fischerleitner, Haindl, Hofmann, Jahoda, Müller, Pann, Paschinger, Straniak, Zothe.

${ }^{21}$ Dabei handelt es sich um: Ambros, Frisch, Gabler, Gassner, Haindl, Hiltscher, Hofmann, Holler, Hollmann, Hotzy, Jahoda, Jedliczka, Karbeutz, Michlmayr, Midlarz, Mühlvenzl, Neubauer, Schuster, Schwarzinger, Weisswasser, Wintersperger.

${ }^{22}$ Dabei handelt es sich um: Bernard, Fellner, Frisch, Fryda, Gabler, Gassner, Hiltscher, Holler, Hotzy, Jahoda, Jedliczka, Kuch, Lahr, Midlarz, Mitterlechner,
} 
mehr als vier Klagen zukamen. Aufgrund dessen kann angenommen werden, dass die Zuteilung der einzelnen Fälle an die Geschäftsabteilungen bzw. die einzelnen Richter nach einem klassischen Muster, z.B. alphabetisch nach dem Namen des Klägers oder Beklagten, erfolgte.

Die Streuung ab 1941 lässt jedoch darauf schließen, dass das System ab diesem Zeitpunkt - zumindest hinsichtlich „ideologischer Fälle“ - abgeändert wurde. Von den 72 Urteilen aus 1941 wurden 23 von LGR Wintersperger ${ }^{23}$ und 23 von LGR Hiltscher ${ }^{24}$ entschieden; bei beiden handelt es sich um politisch verlässliche NS-Richter. ${ }^{25}$ Die übrigen Urteile wurden auf 15 andere Richter verteilt. ${ }^{26}$

Sarauer, Schrom, Seibt, Stahl, Stark, Straniak, Weisswasser, Wintersperger, Zothe.

23 Anton Wintersperger, geboren am 9.5.1881, war schon 1932 der NSDAP beigetreten. Im März 1938 wurde er ans LGZ Wien berufen, wobei er aufgrund seiner langjährigen NSDAP-Mitgliedschaft als "politisch einwandfrei“ eingestuft wurde. Im März 1942 wurde er „zur Erfüllung kriegswichtiger behördlicher Aufgaben vom Wehrdienst freigestellt" und im Juni 1942 wurde ihm das Treudienst-Ehrenzeichen 2. Stufe verliehen (ÖStA/AdR, RJM, Wintersperger, I p 18 W 1696).

${ }^{24}$ Hermann Hiltscher, geboren am 3.7.1903, war seit 5.12.1925 Mitglied der NSDAP. 1938 wurde er Richter am Kreisgericht Korneuburg und ab 1.10.1939 zum Landgerichtsdirektor in Wien. Im April 1943 wurde er zur Wehrmacht eingezogen. Nach Kriegsende wurde er vorerst aus dem Justizdienst entlassen, 1951 jedoch wieder rehabilitiert, so dass er seine Tätigkeit als Richter wieder aufnehmen konnte. 1966 war er am Aufbau der Bundesentschädigungskommission und der Bundesverteilungskommission beteiligt. 1969 wurde ihm das Große Ehrenzeichen für Verdienste um die Republik Österreich verliehen (ÖStA/AdR, BMJ, Hiltscher, 5705/68).

${ }^{25}$ Die Entscheidungen der beiden fanden für diverse $\mathrm{Cg}$-Abteilungen statt. So findet sich Wintersperger als Richter von $1 \mathrm{Cg}, 2 \mathrm{Cg}, 6 \mathrm{Cg}, 7 \mathrm{Cg}, 8 \mathrm{Cg}, 9 \mathrm{Cg}, 11 \mathrm{Cg}$, $12 \mathrm{Cg}, 14 \mathrm{Cg}, 15 \mathrm{Cg}$ und $28 \mathrm{Cg}$, Hiltscher wiederum als Richter von $21 \mathrm{Cg}, 22 \mathrm{Cg}, 23 \mathrm{Cg}, 24 \mathrm{Cg}, 25 \mathrm{Cg}, 26 \mathrm{Cg}$, $28 \mathrm{Cg}, 29 \mathrm{Cg}$.

${ }^{26}$ Dabei handelt es sich um: Ambros, Gabler, Gassner, Haindl, Hollmann, Khol, Krieger, Lahr, Michelmayr, Mitterlechner, Neubauer, Seibt, Schiroky, Stahl, Weisswasser.
Insbesondere ist darauf hinzuweisen, dass die Richter Wintersperger und Hiltscher sich durch einen besonders hohen Prozentsatz an ablehnenden Entscheidungen (circa $85 \%$ ) in solchen „ideologischen Fällen" von den übrigen Richtern (durchschnittlich $25 \%$ ) unterschieden.27 Darüber hinaus findet man gerade bei diesen beiden Richtern ideologische Argumentationen in den Entscheidungsbegründungen.

Diese Tendenz setzte sich in den folgenden Jahren fort, wobei in weiterer Folge die ideologischen Urteile nunmehr mehrheitlich von einem Richter, nämlich LGR Wintersperger, gefällt wurden. Im Jahr 1942 sind 52 von 87 Entscheidungen zur Anfechtung der ,jüdischen“ Abstammung auf LGR Wintersperger zurückzuführen, ${ }^{28} 1943$ entschied derselbe 22 von 33 Fällen. ${ }^{29} 1944$ und 1945 gab es insgesamt nur mehr drei Urteile, ${ }^{30}$ wobei Wintersperger für zwei Entscheidungen und Hiltscher für eine verantwortlich zeichnen. Bemerkenswert ist zudem, dass sich der Anteil der ablehnenden Entscheidungen ab 1942 bei allen Richtern bei ungefähr $50 \%$ einpendelte. ${ }^{31}$

\footnotetext{
${ }^{27}$ Unter den 46 Entscheidungen von Wintersperger und Hiltscher finden sich nur 7 stattgebende Entscheidungen, die allesamt von Wintersperger gefällt wurden. Von 26 Entscheidungen der übrigen Richter wurden 19 zugunsten der klagenden Partei entschieden.

${ }^{28}$ Die übrigen 35 entschieden Judex (x11), Hiltscher (x10), Reiser (x6), Pulpan (x6), Schreyer, Weisswasser. ${ }^{29}$ Die übrigen wurden von Judex (x5), Pulpan ( $\left.x 4\right)$ und jeweils ein Verfahren von Hiltscher und Mühlvenzl entschieden.

301944 wurden die meisten Verfahren entweder aus formellen Gründen zurückgewiesen oder von der klagenden Partei zurückgezogen. Teilweise lässt sich das Verfahrensende aus dem Akt nicht erkennen.

${ }^{31}$ Von den 52 Entscheidungen von Wintersperger wurde in 28 Fällen zugunsten der klagenden Partei entschieden. Unter den 33 Verfahren der übrigen Richter gab es nur 15 stattgebende Urteile.
} 


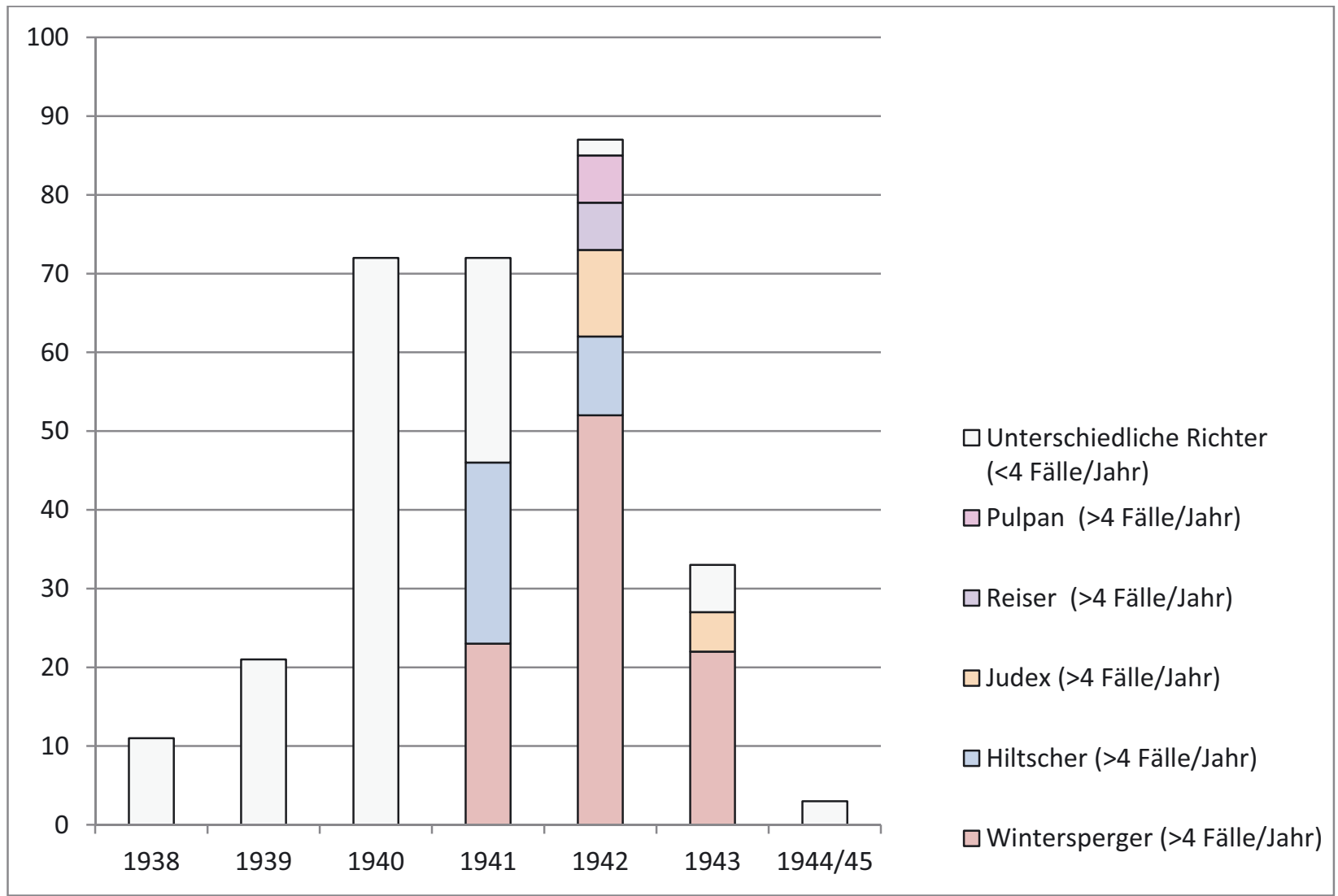

Grafik 2: Richterliche Zuteilung in Verfahren zur ,jüdischen Abstammung“

Die Fälle von Wintersperger und Hiltscher lassen den Schluss zu, dass Nationalsozialisten der ersten Stunde gerade in jenen Bereichen zum Einsatz kamen, die für die Durchsetzung der NS-Ideologie bedeutsam waren. Abschließende Aussagen über die Personalpolitik und die Mo- tive hinter der richterlichen Zuteilung am LGZ/LG Wien lassen sich auf Grundlage dieser Zahlen nicht treffen, da es dafür wohl einer genaueren Aufarbeitung der Biographien aller involvierten Richter bedürfte. 


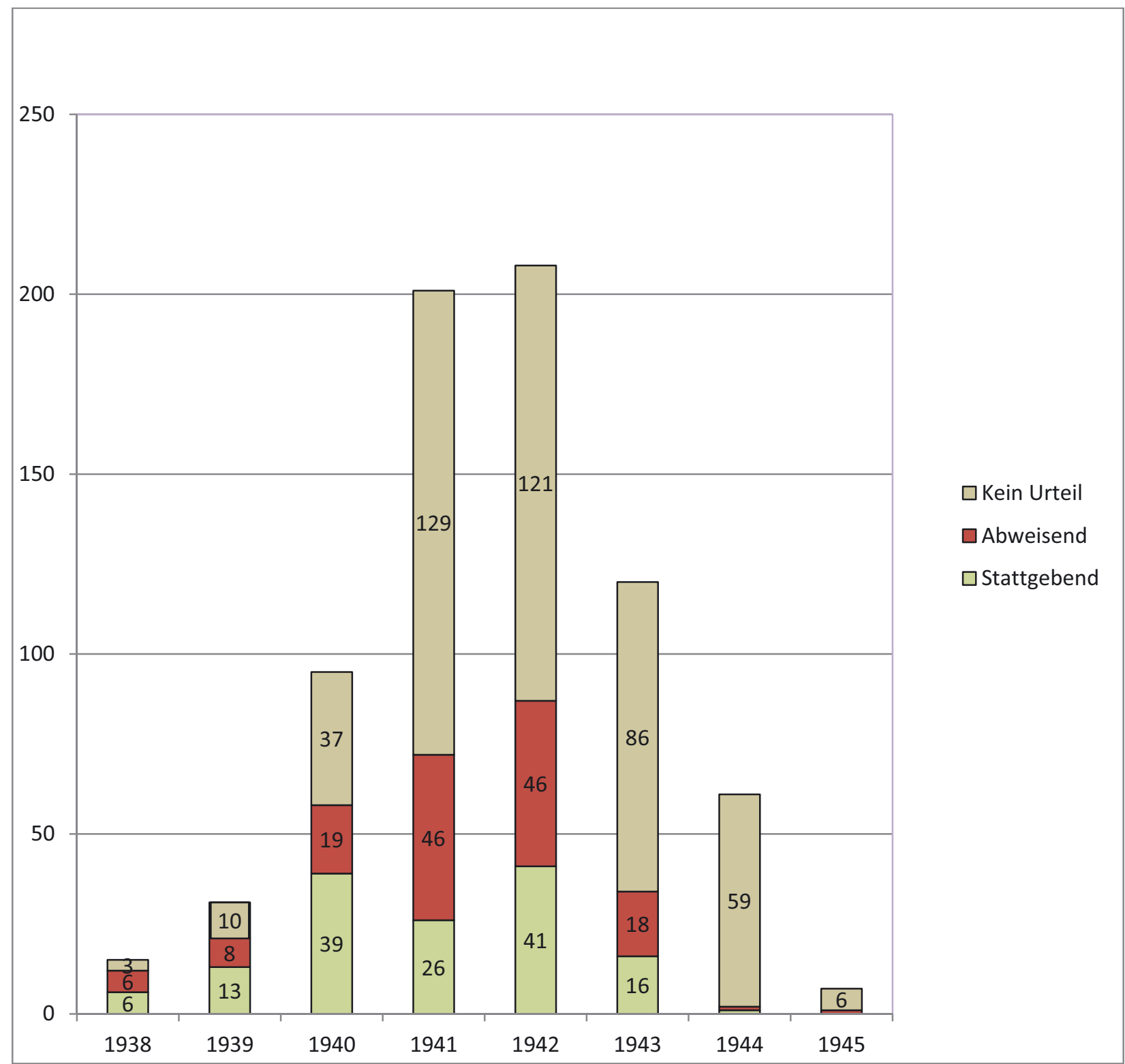

Grafik 3: Die Verfahren zur Anfechtung der ,jüd. Abstammung“ 1938-1945

Es lässt sich jedoch zweifelsfrei feststellen, dass jene Richter, die 1940 durch besonders restriktive Urteile und ideologische Argumentationen gegenüber Klägern ,jüdischer Abstammung“ aufgefallen sind, im darauf folgenden Jahr für einen Großteil solcher Fälle zuständig wurden. Dafür spricht auch eine handschriftliche Anmerkung, die in einem Gerichtsakt aus dem Jahr 1944 gefunden wurde, in der es heißt: „wird dem LGDir Wintersperger abgetreten, da der gesetzliche Vater auf den Klagsausfertigungen Jude ist. “32 Dadurch zeichnet sich das Bild ab, dass vor allem ab 1941 jene Zivilrichter zum Zug kamen, die ihre Rolle als Wahrer "öffentlicher" (im Sinne von nationalsozialistischer) Interessen mit Eifer und Elan erfüllten. ${ }^{33}$

${ }^{32}$ W., Friedrich Marie, Klage (29.4. 1944) WStLA 2.3.5.A24, Kart. 1443/6/43, 6 Cg 110/44.

33 Vgl. Neugebauer, Richter in der NS-Zeit 58f. 


\section{c. Der Einfluss von Parteistellen und Behörden auf das Abstammungsverfahren}

Aus den Gerichtsakten konnten zudem neue Erkenntnisse über die Veränderungen der Umstände gewonnen werden, unter denen Richter am LGZ/LG Wien Entscheidungen trafen. Dabei waren jene Faktoren von Interesse, die die Entscheidung der Richter beeinflussten, insbesondere die Fragestellungen, ob und in welcher Form staatliche Behörden und Parteistellen mit Forderungen an die Gerichte herantraten und inwieweit solche Interventionen Auswirkungen hatten. Aus den Gerichtsakten ließen sich diesbezüglich schriftliche Stellungnahmen diverser Behörden nachweisen. In den meisten Fällen basierte die Miteinbeziehung dieser Behörden auf einer gesetzlichen oder zumindest behördeninternen Anordnung.

\section{Die Reichsstelle für Sippenforschung und das Gauamt für Sippenforschung ${ }^{34}$}

Durch die AV zur Beteiligung der Reichsstelle für Sippenforschung bei erb- und rassekundlichen Untersuchungen vom 10. Februar 193935 wurden die Gerichte dazu angehalten, in Fällen, in denen die "rassische Einordnung" der betroffenen Person indirekt zur Frage stand, die Reichsstelle für Sippenforschung (Reichssippenamt) beizuziehen und die Vermittlung der erforderlichen Untersuchungen von dieser durchführen zu lassen. In Fällen, in denen der Kläger schon einen Abstammungsbescheid beantragt hatte, wurden dieser und die dazugehörigen Unterlagen an das Gericht übersandt. Grundsätzlich wurde das Reichssippenamt somit für die Übermittlung von Beweismitteln herangezogen. In den Gerichtsakten des LGZ/LG Wien ist

\footnotetext{
${ }^{34}$ Dieser Abschnitt entspricht BUKOR, Zivilrecht und Ideologie 212-213.

${ }^{35}$ AV vom 10. 2. 1939, DJ 1939, 350.
}

jedoch auch ein Fall erhalten, in dem es eine darüber hinausgehende Stellungnahme abgab:

In dem betreffenden Verfahren hatte der Kläger parallel zur zivilrechtlichen Abstammungsklage eine verwaltungsrechtliche „rassische Einordnung" beantragt. In der Regel wartete das Reichssippenamt in einer solchen Situation die Entscheidung des Zivilgerichts ab, in diesem Fall wusste man dort jedoch nichts von der zivilrechtlichen Klage. Da es sich bei dem Kläger um einen Rechtsanwalt handelte, dessen Publikationen in nationalen Kreisen geschätzt wurden, war man anscheinend darauf bedacht, eine besonders „sorgfältige“ Prüfung durchzuführen. So intervenierte der Direktor des Reichssippenamtes insofern, als er das Gericht bat, sich in der Beweisaufnahme auf den positiven Abstammungsbescheid des Reichssippenamtes zu stützen und der Klage stattzugeben. ${ }^{36}$ Das Verfahren wurde jedoch nicht durch eine gerichtliche Entscheidung beendet. Der Kläger zog die Klage am 7. März 1943 aufgrund "gesundheitlicher Behinderung und kriegsbedingter Verhältnisse" unter dem Vorbehalt der Wiedereinbringung zurück. ${ }^{37}$

\section{Die Zentralstelle für jüdische Auswanderung ${ }^{38}$}

Ab 1941 lässt sich auch die Miteinbeziehung der Zentralstelle für jüdische Auswanderung nachweisen, die durch das Polizeipräsidium über Verfahren informiert wurde, in denen eine ,jüdische Abstammung" angefochten wurde. ${ }^{39}$ Bis zu ihrer Schließung im März 1943 gab die Zent-

\footnotetext{
${ }^{36}$ Reichssippenamt (8.4.1942) WStLA 2.3.5.A24, Kart. 203/6/41, 6 Cg 268/41.

${ }^{37}$ L., Victor, Klagsrückziehung (7.3. 1943) WStLA 2.3.5.A24, Kart. 203/6/41, 6 Cg 268/41.

${ }^{38}$ Dieser Abschnitt entspricht BUKOR, Zivilrecht und Ideologie 213-216.

${ }^{39}$ Der Hinweis, dass die ZStfjA vom Polizeipräsidium eingebunden wurde, findet sich teilweise in den Mitteilungen der ZStfjA. (ZStfjA, Mitteilung an LG Wien (11. 8. 1942) WStLA 2.3.5.A24, Kart. 1284/30/39, 30 Cg 371/39; ZStfjA, Mitteilung an LG Wien (3. 8. 1942) WStLA 2.3.5.A24, Kart. 531/14/41, 14 Cg 346/41.
} 
ralstelle mindestens 82 Stellungnahmen ab. In mehreren Fällen wurde dadurch mit Nachdruck gegen ein dem Klagebegehren stattgebendes Urteil Stellung bezogen, um ein „Einschleichen jüdischen Blutes in den deutschen Volkskörper" zu verhindern. ${ }^{40} \mathrm{Zu}$ diesem Zweck wurde die Glaubwürdigkeit des Klagebegehrens bestritten, ${ }^{41}$ zum Teil unter Heranziehung rein speku-

${ }^{40}$ Z.B.: ZStfjA, Mitteilung an LG Wien (8. 8. 1942) WStLA 2.3.5.A24, Kart. 161/5/41, 5 Cg 281/41; ZStfjA, Mitteilung an LG Wien (11. 8. 1942) WStLA 2.3.5.A24, Kart. 1284/30/39, 30 Cg 371/39; ZStfjA, Mitteilung an LG Wien (3. 8. 1942) WStLA 2.3.5.A24, Kart. 531/14/41, 14 Cg 346/41; ZStfjA, Mitteilung an LG Wien (31. 10. 1942) WStLA 2.3.5.A24, Kart. 531/14/41, $14 \mathrm{Cg}$ 351/41; ZStfjA, Mitteilung an LG Wien (29. 7. 1942) WStLA 2.3.5.A24, Kart. 1006/23/41, $23 \mathrm{Cg}$ 316/41; ZStfjA, Mitteilung an LG Wien (7. 8. 1942) WStLA 2.3.5.A24, Kart. 1190/28/41, 28 Cg 231/41; ZStfjA, Mitteilung an LG Wien (30.7. 1942) WStLA 2.3.5.A24, Kart. 21/1/42, 1 Cg 28/42; ZStfjA, Mitteilung an LG Wien (3. 8. 1942) WStLA 2.3.5.A24, Kart. 120/4/42, 4 Cg 40/42; ZStfjA, Mitteilung an LG Wien (1.3. 1943) WStLA 2.3.5.A24, Kart. 122/4/42, 4 Cg 101/42.

${ }^{41}$ ZStfjA, Mitteilung an LG Wien (8. 8. 1942) WStLA 2.3.5.A24, Kart. 161/5/41, 5 Cg 281/41; ZStfjA, Mitteilung an LG Wien (11.8. 1942) WStLA 2.3.5.A24, Kart. 1284/30/39, 30 Cg 371/39; ZStfjA, Mitteilung an LG Wien (3. 8. 1942) WStLA 2.3.5.A24, Kart. 531/14/41, 14 Cg 346/41; ZStfjA, Mitteilung an LG Wien (30. 7. 1942) WStLA 2.3.5.A24, Kart. 21/1/42, 1 Cg 28/42; ZStfjA, Mitteilung an LG Wien (5. 3. 1943b) WStLA 2.3.5.A24, Kart. 255d/7/43, 7 Cg 178/43; ZStfjA, Mitteilung an LG Wien (23. 3. 1943) WStLA 2.3.5.A24, Kart. 1259/29/43, 29 Cg 27/43; ZStfjA, Mitteilung an LG Wien (26.2.1942) WStLA 2.3.5.A24, Kart. 417/11/41, $11 \mathrm{Cg}$ 13/41; ZStfjA, Mitteilung an LG Wien (4. 7. 1942) WStLA 2.3.5.A24, Kart. 913/21/41, 21 Cg 215/41; ZStfjA, Mitteilung an LG Wien (25. 1. 1943) WStLA 2.3.5.A24, Kart. 1096/25/41, 25 Cg 235/41; ZStfjA, Mitteilung an LG Wien (3. 8. 1942) WStLA 2.3.5.A24, Kart. 1188/28/41, 28 Cg 147/41; ZStfjA, Mitteilung an LG Wien (13. 2. 1943) WStLA 2.3.5.A24, Kart. 252/7/42, 7 Cg 160/42; ZStfjA, Mitteilung an LG Wien (16. 2. 1943) WStLA 2.3.5.A24, Kart. 531/14/41, 14 Cg 2/42; ZStfjA, Mitteilung an LG Wien (8. 8. 1942) WStLA 2.3.5.A24, Kart. 958/22/42, 22 Cg 96/42; ZStfjA, lativer Argumente, wie bspw. dass sich die klagende Partei durch ihre Flucht „zum Judentum bekannt" hätte oder dass der unbekannte Aufenthalt der Person beweise, dass sie "Jüdin" sei. ${ }^{42}$

Auch laienhafte Vorstellungen jüdischer Kultur und religiöser Normen wurden von der Zentralstelle für jüdische Auswanderung herangezogen, um das klägerische Vorbringen zu diskreditieren.

Die 1871 geborene Klägerin, die urkundlich das Kind eines ,jüdischen" Ehepaares war, versuchte ihre Abstammung mit dem Hinweis auf ein außereheliches Verhältnis der Mutter mit einem „Arier" zu begründen. Die Zentralstelle verwarf diese Möglichkeit mit dem Argument der an-

Mitteilung an LG Wien (16. 1. 1943) WStLA 2.3.5.A24, Kart. 962/22/42, 22 Cg 238/42; ZStfjA, Mitteilung an LG Wien (9.3.1943) WStLA 2.3.5.A24, Kart. 1008/23/42, 23 Cg 58/42; ZStfjA, Mitteilung an LG Wien (17.9.1942) WStLA 2.3.5.A24, Kart. 1138/26/42, 26 Cg 181/42; ZStfjA, Mitteilung an LG Wien (15.8.1942) WStLA 2.3.5.A24, Kart. 1196/28/42, 28 Cg 120/42; ZStfjA, Mitteilung an LG Wien (14.8.1942) WStLA 2.3.5.A24, Kart. 1254/29/42, 29 Cg 133/42; ZStfjA, Mitteilung an LG Wien (5.3.1943a) WStLA 2.3.5.A24, Kart. 255d/7/43, 7 Cg 178/43.

42 ZStfjA, Mitteilung an LG Wien (11. 8. 1942) WStLA 2.3.5.A24, Kart. 1284/30/39, 30 Cg 371/39; ähnliche Argumentation bei ZStfjA, Mitteilung an LG Wien (23. 2. 1943) WStLA 2.3.5.A24, Kart.375/10/41, 10 Cg 333/41; ZStfjA, Mitteilung an LG Wien (1.3. 1943) WStLA 2.3.5.A24, Kart. 122/4/42, 4 Cg 101/42, ZStfjA, Mitteilung an LG Wien (31. 10.1942) WStLA 2.3.5.A24, Kart. 531/14/41, 14 Cg 351/41; in einem anderen Fall war der Kläger deportiert worden. Der Leiter der Zentralstelle, Alois Brunner, behauptete jedoch in der Stellungnahme, dass der Kläger vor seiner Deportation über seinen Abstammungsprozess befragt worden wäre und dabei „höhnisch“ mitgeteilt hätte, ,er wäre wohl kein Patentanwalt gewesen, wenn er nicht versuchen würde mittels eines Prozesses Mischling zu werden." Daraus wurde geschlossen, dass der Kläger seine Abstammung von einem "Arier" nur erfunden hätte (ZStfjA, Mitteilung an LG Wien [7. 8. 1942] WStLA 2.3.5.A24, Kart. 531/14/41, 14 Cg 1/42). 
geblichen Abgeschlossenheit jüdischen Lebens in Böhmen und Mähren um 1870 und bezeichnete den Inhalt des Vorbringens als „roman- und märchenhaft" ${ }^{43}$ In der Sichtweise der Zentralstelle wäre nicht einmal „ein Prozent von den jetzt vorhandenen Seitensprüngen anzunehmen", da die jüdische Religion einer Jüdin den Seitensprung verbiete. Diese Behauptung versuchte die Zentralstelle mit einer angeblich von „jüdischen Wissenschaftlern“ getätigten Aussage zu untermauern, wonach "nicht $1 \%$ der jetzt auftauchenden Seitensprünge Tatsachen sind". Denn wenn „[e]in Volljude“ wüsste, dass seine Frau „ein Kind von einem Arier“ geboren hätte, würde er „niemals die Ehe weiter aufrecht erhalten und das Kind eines Christen betreuen und pflegen". ${ }^{4}$

Solche Bezugnahmen auf religiöse Regeln des Judentums finden sich auch in anderen Verfahren. So stritt die Zentralstelle bspw. die Glaubwürdigkeit eines Ehebruchs der Mutter mit dem Argument ab, dass ,"der Jude seine Ehefrau dem Talmud entsprechend, weil sie sich mit einem Goi eingelassen hat", mit Sicherheit verlassen hätte. ${ }^{45}$

Von Seiten der Zentralstelle blieb es nicht nur bei solchen Versuchen, auf die richterlichen Entscheidungen in einem laufenden Verfahren

\footnotetext{
${ }^{43}$ Mit einer fast gleichlautenden Formulierung (,,roman- und märchenhaft") stritt die Zentralstelle auch eine andere Klage ab. Hier brachte sie vor, dass um 1860 das jüdische Leben in Ungarn derart abgeschlossen gewesen wäre, dass nicht angenommen werden könne, dass ein ungarischer Magnat ein ehebrecherisches Verhältnis mit einer „volljüdischen“ Frau unterhalten hätte (ZStfjA, Mitteilung an LG Wien [29. 7. 1942] WStLA 2.3.5.A24, Kart. 1135/26/42, 26 Cg 79/42).

${ }^{44}$ ZStfjA, Mitteilung an LG Wien (30.7. 1942) WStLA 2.3.5.A24， Kart. 1247/29/41， 29 Cg 271/41; ähnliche Argumentation auch bei ZStfjA, Mitteilung an LG Wien (17. 9. 1942) WStLA 2.3.5.A24, Kart. 1138/26/42, 26 Cg 181/42.

${ }^{45}$ ZStfjA, Mitteilung an LG Wien (29. 7. 1942) WStLA 2.3.5.A24, Kart. 1135/26/42, 26 Cg 79/42.
}

gezielt Einfluss $\mathrm{zu}$ nehmen. Darüber hinaus ergingen von ihr auch mehrmals Aufforderungen an das Gericht, im Fall des Erscheinens der klagenden Partei sofort Meldung zu erstatten. Als diesbezügliche Begründung wurde angeführt dass die betreffende Partei versuche, sich der „Evakuation“ zu entziehen. ${ }^{46}$ Über das Verhalten der Richter hinsichtlich solcher Anordnungen geben die vorliegenden Akten keine Auskunft.

Auch wenn sich direkte Auswirkungen von Stellungnahmen der Zentralstelle auf die Rechtsprechung des LGZ/LG Wien schwerlich nachweisen lassen, ist doch augenscheinlich, dass von jenen Verfahren, in denen sich die Zentralstelle gegen ein positives Urteil aussprach, nur ein einziges mit einem stattgebenden Urteil endete. ${ }^{47}$ In diesem Fall wurde das Klagebegehren zwar zuerst abgewiesen, die Entscheidung jedoch vom RG aufgehoben und zurückverwiesen. Erst nach der Zurückverweisung gab das LG Wien der Klägerin Recht, obwohl sich die ZStfjA inzwischen gegen eine positive Entscheidung ausgesprochen hatte. ${ }^{48}$ Andererseits kam es auch nur in insgesamt vier Verfahren zu einer Abweisung der Klage. In den anderen Fällen wurde ein dem Klagebegehren entsprechendes Urteil jedoch verhindert, weil die klagende Par-

\footnotetext{
${ }^{46}$ Z.B. ZStfjA, Mitteilung an LG Wien (19. 2. 1943) WStLA 2.3.5.A24, Kart. 1187/28/41, 28 Cg 113/41.

${ }^{47}$ In einem weiteren Verfahren, in dem das erstinstanzliche Urteil das Klagebegehren abgewiesen hatte, entschied das OLG Wien trotz widersprechender Aufforderung der ZStfjA zugunsten der Klägerin (OLG Wien 1. 10. 1942, 2 R 224/42).

${ }^{48}$ LG Wien 15. 1. 1944, 2 Cg 15/42 (Wintersperger): Die ZStfjA betonte, dass es sich bei der in die Schweiz geflohenen Klägerin um eine Verwandte des Juden Heinrich Heine handle und dem "Versuch, sich in den deutschen Volkskörper hineinzuschwindeln" umso mehr entgegen getreten werden müsse (ZStfjA, Mitteilung an LG Wien [21. 9. 1942] WStLA 2.3.5.A24, Kart. 48/2/41, 2 Cg 15/42).
} 
tei während des Verfahrens verstorben, ${ }^{49}$ deportiert, ${ }^{50}$ ermordet ${ }^{51}$ oder geflohen ${ }^{52}$ war bzw. die Klage zurückziehen musste. ${ }^{53}$ Mangels konkreter Anhaltspunkte kann keine Aussage darüber getroffen werden, ob die Zentralstelle Teile der Richterschaft am LGZ/LG Wien durch Drohungen unter Druck setzte, wie sie es bei „unbequemen" Rechtsanwälten ganz offen zu tun pflegte. ${ }^{4}$ Ihren Abbruch erfuhren die Stellungnahmen durch die Auflösung der Zentralstelle im Frühjahr 1943. 55

\section{Der Staatsanwalt und der Oberreichsanwalt ${ }^{56}$}

Mit dem Gesetz über die Mitwirkung des Staatsanwalts in bürgerlichen Rechtssachen vom

\footnotetext{
${ }^{49}$ M., Antonie, Anzeige über Vereinbarung des Ruhens des Verfahrens (4.11. 1942) WStLA 2.3.5.A24, Kart. 1006/23/41, 23 Cg 316/41.

${ }^{50}$ ZStfjA, Mitteilung an LG Wien (4. 8. 1942) WStLA 2.3.5.A24, Kart. 1007/23/41, 23 Cg 19/42; ZStfjA, Mitteilung an LG Wien (7.8.1942) WStLA 2.3.5.A24, Kart. 1190/28/41, 28 Cg 231/41.

${ }^{51}$ ZStfjA, Mitteilung an LG Wien (3. 8. 1942) WStLA 2.3.5.A24, Kart. 120/4/42, 4 Cg 40/42.

${ }^{52}$ ZStfjA, Mitteilung an LG Wien (23. 1. 1943) WStLA 2.3.5.A24, Kart. 244/7/41, 7 Cg 135/41; ZStfjA, Mitteilung an LG Wien (3.11.1942) WStLA 2.3.5.A24, Kart. 532/14/42, 14 Cg 13/42.

${ }^{53}$ LG Wien, Übertragung des Kurzschriftenprotokolls (2. 8. 1944) WStLA 2.3.5.A24, Kart. 1196/28/42, $28 \mathrm{Cg} 120 / 42$.

${ }^{54}$ In Fällen, in denen die betroffene Person deportiert oder geflohen war, drohte die ZStfjA dem Rechtsvertreter der klagenden Partei mit einer Anzeige wegen „Behördenbelästigung", wenn diese weitere Schritte ergreifen sollte, um das Verfahren fortzusetzen: ZStfjA, Mitteilung an LG Wien (4.8.1942) WStLA 2.3.5.A24, Kart. 1007/23/41, 23 Cg 19/42; ZStfjA, Mitteilung an LG Wien (7.8. 1942) WStLA 2.3.5.A24, Kart. 1190/28/41, 28 Cg 231/41. In beiden Fällen handelte es sich um den Rechtsanwalt Dr. Fritz Habietinek, der von der Zentralstelle als "Judenmischling" beschrieben wurde, der Abstammungsprozesse „inszeniere".

${ }^{55}$ ANDERL, RUPNOW, WENCK, Zentralstelle für Jüdische Auswanderung 293.

${ }^{56}$ Dieser Abschnitt entspricht BUKOR, Zivilrecht und Ideologie 216-219.
}

15. Juli $1941^{57}$ und dessen Spezifizierung durch die amtliche Begründung vom 22. Juli $1941^{58}$ waren Staatsanwälte befugt, in laufenden ordentlichen Zivilprozessen am Verfahren teilzunehmen, Tatsachen und Beweise vorzubringen und sich zur erlassenen Entscheidung gutachtlich zu äußern. Daher wurde die Verständigung der Staatsanwaltschaft auch ein standardisiertes Prozedere im Rahmen des Verfahrens. ${ }^{59}$ Die praktische Auswirkung dieses Gesetzes scheint sich jedoch in Grenzen gehalten zu haben, da sich in keinem der betroffenen Verfahrensakten Beweisanträge oder Stellungnahmen des Staatsanwalts finden. In der Praxis relevant war jedoch nachweislich die Möglichkeit des Staatsanwalts, nach Fristablauf eine Ehelichkeitsanfechtung einzubringen, die durch die Verordnung über die Angleichung familienrechtlicher Vorschriften vom 6. Februar $1943^{60}$ eingeführt wurde. ${ }^{61}$

Zudem ermöglichte das Gesetz vom 15. Juli 1941 auch ein Einschreiten des Oberreichsanwalts gegen jedes rechtskräftig entschiedene Zivilverfahren. Bei schweren Bedenken gegen die Richtigkeit der Entscheidung konnte er binnen eines Jahres ab Eintritt der Rechtskraft einen Wiederaufnahmeantrag beim Reichsgericht einreichen (§ 2). Zweifellos steht fest, dass diese Regelung eine Beschränkung der richterlichen Unabhän-

\footnotetext{
${ }^{57}$ RGBl. I 1941, 383.

${ }^{58}$ Amtliche Begründung zum Gesetz über die Mitwirkung des Staatsanwalts in bürgerlichen Rechtssachen vom 22. 7. 1941, DJ 1941, 791.

${ }^{59}$ Siehe Formblatt, z.B. LG Wien, Formblatt (1. 7. 1942) WStLA 2.3.5.A24, Kart. 1284/30/39, 30 Cg 371/39.

${ }^{60}$ RGBI. I 1943, 80.

${ }^{61}$ Vgl. z.B.: Oberstaatsanwalt beim Landgericht Wien, Klage wegen Bestreitung der Ehelichkeit (27. 5. 1943) WStLA 2.3.5.A24, Kart. 212c/6/43, 6 Cg 143/43; Oberstaatsanwalt beim Landgericht Wien, Klage wegen Feststellung der Unehelichkeit (27.3. 1943) WStLA 2.3.5.A24, Kart. 294b/8/43, 8 Cg 77/43; Oberstaatsanwalt beim Landgericht Wien, Klage wegen Bestreitung der ehelichen Geburt (4.6.1943) WStLA 2.3.5.A24, Kart. 452c/12/43, 12 Cg 145/43.
} 
gigkeit und eine Einflussnahme des Staates auf die Justiz intendierte und damit wohl auch Unbehagen bei der Richterschaft auslöste. ${ }^{62}$ In dem dieser Untersuchung zugrunde liegenden Fundus an Entscheidungen lässt sich jedoch kein merkbarer Anstieg an Wiederaufnahmeverfahren ausmachen.

Erst die Einführung der Verordnung über die Wiederaufnahme rechtskräftig entschiedener Abstammungsklagen vom 27. Jänner $1944^{63}$ erhöhte die Anzahl von Wiederaufnahmeverfahren signifikant. Der Oberreichsanwalt konnte nunmehr beim Reichsgericht beantragen, rechtskräftig entschiedene Verfahren zur Feststellung der Abstammung von Juden und ,jüdischen" Mischlingen, durch die sich die rassistisch zugeschriebene Qualifikation dieser Person verändert hatte, wieder aufzunehmen. Im Gegensatz zum Gesetz vom 15. Juli 1941 war ein solcher Antrag auch nach Ablauf der vorgesehenen Frist von einem Jahr möglich. Auf welcher Grundlage die Auswahl der Urteile erfolgte, lässt sich nicht genau sagen; zumindest in einzelnen Fällen ist das Gericht nachweislich von sich aus mit Kritik an bereits ergangenen Urteilen an die Staatsanwaltschaft herangetreten. ${ }^{64}$

Primär kam es bei Abstammungsverfahren, in denen Kinder erfolgreich ihre NichtAbstammung von einem ,jüdischen“ Erzeuger geltend gemacht hatten, zur Prüfung eines Wie-

\footnotetext{
${ }^{62}$ DAUT, NS-Einfluß auf die Rechtsprechung 204-207.

${ }^{63}$ RGBl. I 1944, 52.

${ }^{64}$ LG Wien, Mitteilung an Staatsanwaltschaft beim OLG Wien (23.5.1944) WStLA 2.3.5.A24, Kart. 444/12/38, 12 Cg 220/38 (Hofmann, A.); LG Wien, Mitteilung an Staatsanwaltschaft beim OLG Wien (24.5.1944) WStLA 2.3.5.A24, Kart. 1166/28/39, 28 Cg 121/39; LG Wien, Mitteilung an Staatsanwaltschaft beim OLG Wien (15.2. 1945) WStLA 2.3.5.A24, Kart. 1097/25/41, 25 Cg 241/41; LG Wien, Mitteilung an Staatsanwaltschaft beim OLG Wien (15.2. 1945) WStLA 2.3.5.A24, Kart. 1240/29/41, 29 Cg 43/41.
}

deraufnahmeantrages. ${ }^{65}$ Manchmal blieben diese im Untersuchungsstadium stecken, ${ }^{66}$ in vielen Fällen wurde der Antrag jedoch eingebracht. Zur Wiederaufnahme von Abstammungsverfahren kam es vor allem in solchen Fällen, in denen sich die Urteile hauptsächlich auf Zeugenaussagen stützten. ${ }^{67}$

Je nach beanstandetem Mangel konnte das Verfahren aufgrund eines erfolgreichen Wiederaufnahmeantrages entweder zur erneuten Verhandlung und Entscheidung an die Vorinstanz zurückverwiesen werden ${ }^{68}$ oder das Gericht entschied - wenn das Verfahren zur Entscheidung reif war oder die Klage verfahrensrechtlich als unzulässig erkannt wurde - in der Sache selbst.

In einem Wiederaufnahmeverfahren des Jahres 1944, in dem der Oberreichsanwalt verfahrensrechtliche Mängel vorbrachte, argumentierte das

${ }^{65}$ In einzelnen Fällen brachte der Oberreichsanwalt auch Anträge ein, obwohl der Fall keinen „rassischen" Hintergrund hatte (vgl. RG 30. 8. 1944, VII 101/44); hierbei galt jedoch das Gesetz über die Mitwirkung des Staatsanwalts in bürgerlichen Rechtssachen aus 1941 als Rechtsgrundlage und der Staatsanwalt war an die einjährige Frist ab Rechtskraft des Urteils gebunden.

${ }^{66}$ Generalstaatsanwalt, Mitteilung an LG Wien (10. 10. 1944) WStLA 2.3.5.A24, Kart. 150/5/40, 5 Cg 49/40; Generalstaatsanwalt, Mitteilung an LGR Wintersperger (27.11. 1944) WStLA 2.3.5.A24, Kt. 905/21/40, 21 Cg 305/40; Generalstaatsanwalt, Mitteilung an LG Wien (7.3. 1945) WStLA 2.3.5.A24, Kart. 686/17/41, 17 Cg 57/41.

${ }^{67}$ Vgl. RG 30. 8. 1944, VII 109/44; RG 30. 8. 1944, VII 120/44; RG 13. 9. 1944, VII 129/44; RG 4. 10.1944, VII 144/44; RG 18.10.1944, VII 131/44; RG 18.10.1944, VII 149/44; RG 18.11.1944, VII 151/44; RG 18. 11. 1944, VII 162/44; RG 29. 11. 1944, VII 168/44. ${ }^{68}$ Vgl. RG 30. 8. 1944, VII 120/44; RG 13. 9. 1944, VII 129/44; RG 18.11.1944, VII 151/44; RG 25.11.1944, VII 166/44; RG 29.11.1944, VII 168/44; RG 29. 11. 1944, VII 168/44; RG 2. 12. 1944, VII 182/44; RG 6. 1. 1945, VII 208/44; z.B. mit der Forderung, ein erbbiologisches Gutachten durchzuführen: RG 17. 10. 1944, VII 143/1944; im Geltungsbereich des ABGB, vgl. RG 4. 10. 1944, VII 144/44; RG 18. 10. 1944, VII 149/44; RG 18. 11. 1944, VII 162/44; teilweise Abweisung RG 29. 11. 1944, VII 168/44. 
Reichsgericht damit, dass eine Fortsetzung des Verfahrens gegen die Beklagten nicht möglich sei und „mit der Abweisung der Klage die Interessen der Volksgemeinschaft vorläufig gewahrt" wären. ${ }^{69}$

Aus dem im Rahmen der Untersuchung analysierten Bestand geht hervor, dass Wiederaufnahmeanträge des Oberreichsanwaltes ausnahmslos im Sinne des Wiederaufnahmeantrages entschieden wurden. ${ }^{70}$

In einem Fall heiratete die Mutter im Jahre 1897, elf Jahre nach der Geburt des unehelichen Kindes, einen "Juden", der das Kind legitimierte. Die Tochter klagte im Jahre 1939 auf Feststellung, dass ihr Vater eine andere Person sei als ihr gesetzlicher Vater, nämlich ein „Arier“. Ihr tatsächlicher Vater sei jedoch nach Argentinien ausgewandert und bereits gestorben. Die Klage stützte sich auf Aussagen des Onkels und des Ehemannes der Klägerin, die Informationen von der bereits verstorbenen Mutter erhalten hatten. Die Behauptung der Zeugen, dass die Mutter und der genannte Vater zur kritischen Zeit im gemeinsamen Haushalt gelebt hatten, konnte durch die polizeilichen Meldedaten nicht bestätigt werden. Der legitimierende Vater war zu dieser Zeit auch nicht im Haushalt der Mutter gemeldet, jedoch kurze Zeit später, noch vor der Geburt der Klägerin. Das daraufhin durchgeführte anthropologische Gutachten fand bei der Klägerin keine "Merkmale des für das jüdische Volk kennzeichnenden vorderasiatischorientalischen Rassengemisches". Auf dieser Grundlage hatte das Landgericht Wien zugunsten der Klägerin entschieden. Den Wiederaufnahmeantrag des Oberreichsanwalts sah das Reichsgericht als begründet, weil den Zeugen-

\footnotetext{
${ }^{69}$ RG 13. 12. 1944, VII 188/44.

${ }^{70}$ Vgl. RG 17. 10. 1944, VII 143/1944; RG 30. 8. 1944, VII 119/44; RG 30. 8. 1944, VII 101/44; RG 18. 10. 1944, VII 149/44; RG 18.11.1944, VII 151/44; RG 18. 11. 1944, VII 162/44; RG 25. 11. 1944, VII 166/44; RG 2. 12. 1944, VII 182/44; RG 6. 1. 1945, VII 208/44.
}

aussagen alleine keine Beweiskraft zuerkannt werden konnte. Die Existenz des "arischen“ Vaters und sein Zusammenleben mit der Kindesmutter konnte dadurch nicht sicher nachgewiesen werden. Darüber hinaus konnte das erstellte Gutachten in der Abstammungsfrage nicht die an Sicherheit grenzende Wahrscheinlichkeit erbringen, die notwendig wäre, um die Vaterschaft des gesetzlichen Vaters auszuschließen. Daher wurde die Entscheidung aufgehoben und zurückgewiesen. ${ }^{71}$

\section{Weitere Parteistellen und Behörden, die am Verfahren beteiligt waren ${ }^{72}$}

In den Gerichtsakten finden sich spätestens $a b$ 1942 Beschlüsse, die den Rückschluss erlauben, dass das Gericht eine Vielzahl von Einrichtungen in das Beweisverfahren miteinbezog. ${ }^{73}$ Dazu gehörten neben dem Reichssippenamt auch das Gauamt für Sippenforschung (Gausippenamt), das Polizeipräsidium und das Meldeamt. Die Auskünfte dieser Stellen bezogen sich meist auf Meldedaten oder Berichte über etwaige Abstammungsverfahren des Klägers sowie seines familiären Umfeldes. Im Rahmen der Einbeziehung des Polizeipräsidiums kam es teilweise auch zu Stellungnahmen der Kriminalpolizeileitstelle sowie der Gestapo. Diese übermittelten bspw. Informationen über die politische Einstellung der klagenden Partei ${ }^{74}$ oder ihre „rassische Einordnung “. ${ }^{\text {. }}$

${ }^{71}$ RG 2. 12. 1944, VII 182/44; aufgehoben wurden auch andere Urteile, die sich auf ein Gutachten stützten, in dem festgestellt wurde, dass keine Merkmale der „semitischen Rasse“ am Kläger bzw. an der Klägerin festgestellt werden konnten: RG 25.11.1944, VII 159/44; RG 25. 11. 1944, VII 166/44; RG 6.1.1945, VII 208/44.

72 Dieser Abschnitt entspricht BUKOR, Zivilrecht und Ideologie 219-220.

${ }^{73}$ LG Wien, Formblatt (1. 7. 1942) WStLA 2.3.5.A24, Kart. 1284/30/39, 30 Cg 371/39.

${ }^{74}$ So wurde in einem Fall über die Klägerin berichtet, dass gegen ihre Aufnahme in den Reichsverband der deutschen Presse keine staats- und abwehrpolizeili- 
In einem Verfahren aus dem Jahre 1941 versuchte die Klägerin, deren Mutter „Arierin“ war, die Abstammung von dem ,jüdischen“ Ehemann der Mutter anzufechten, um ihren Sohn vor der "rassischen Einordnung" als "Jude" zu schützen. Pikanterweise war ihr Sohn schon in den 1920er Jahren für die NSDAP tätig geworden. Der Brief der Gestapo ging auf diesen „merkwürdigen" Umstand ein, und kommentierte dies damit, dass es sich „um einen jener nicht allzu seltenen Fälle von jüdischem Antisemitismus handelt". Abgesehen von dem Bericht über das laufende Abstammungsverfahren des Sohnes vor dem LGZ Innsbruck, wurde in dem Bericht darauf hingewiesen, dass „die Taktik seiner Verteidigung und sein ganzes Benehmen [...] ein echt jüdisches Gebahren“ und eine „nur mit überwiegend jüdischem Blutsanteil zu erklärende Raffiniertheit" aufweise. „[O]hne zu versuchen, auf das dort laufende Verfahren irgendwie Einfluss nehmen zu wollen," teilte der Gestapo-Beamte freimütig in dem Schreiben an das LG Wien mit, „darf ich zur Information noch darauf hinweisen, dass [der Kläger] hier

chen Bedenken bestanden und, dass sie der NSDAP in politischer Hinsicht schon lange nahe stehe: Gestapo, Mitteilung an LG Wien (16.1.1941) WStLA 2.3.5.A24, Kart. 912/21/41, 21 Cg 173/41.

${ }^{75}$ Der Kläger war in Gestapo-Haft gewesen, jedoch während einer anthropologischen Untersuchung geflüchtet. Daher wurde er von der Gestapo als Jude behandelt und mit Schutzhaft und Überstellung in ein KZ bedroht (Gestapo, Mitteilung an LG Wien [6. 8. 1943] WStLA 2.3.5.A24, Kart. 294d/8/43, 8 Cg 168/43); die Kriminalpolizeistelle hatte diverse Personen aus dem Umfeld des in Haft befindlichen Klägers befragt, die seiner Behauptung, dass er nicht von seinen gesetzlichen Eltern abstamme, widersprachen. Die Kriminalpolizeistelle ordnete ihn daher als "Volljuden" ein und bezeichneten seine Angaben abfällig als „jüdischen Dreh“ (Kriminalpolizeistelle Wien [Dienststelle Wieden], Mitteilung an LG Wien [9. 5. 1942] WStLA 2.3.5.A24, Kart. 694/17/42, 17 Cg 23/42). als einwandfreier Jude angesehen wird."76 $\mathrm{Ob}$ dieses Schreiben einen nachhaltigen Einfluss auf das Verfahren gehabt hätte, lässt sich in diesem Fall nicht feststellen, da das Verfahren mangels einer gaurechtsamtlichen Vertretungsbefugnis des Anwaltes zurückgewiesen wurde. ${ }^{77}$

\section{d. Die Entwicklung der Beweismittel ${ }^{78}$}

Die Feststellung der Nicht-Abstammung vom vermuteten Vater verlangte gemäß §§158, 159 ABGB den Nachweis der Unmöglichkeit, vom vermuteten Vater gezeugt worden $\mathrm{zu}$ sein. ${ }^{79}$ Bei ehelichen Kindern bedeutete dies in der Praxis jedoch, dass die Zeugung durch den gesetzlichen bzw. vermuteten Vater mit einer an Sicherheit grenzenden Wahrscheinlichkeit ausgeschlossen werden musste. ${ }^{80}$ Die Entscheidung, welche Beweismittel für diesen Nachweis vorgebracht werden sollten, oblag vor 1938 im Wesentlichen den Parteien; dem Gericht blieb gemäß § 275 ZPO jedoch vorbehalten, Beweismittel mit Beschluss zurückzuweisen, wenn es diese für unerheblich hielt. Nach dem "Anschluss" kam es einerseits durch unmittelbare öffentliche Einflussnahme, andererseits aber auch durch ideologisch motivierte Eigeninitiative der Richter zu wesentlichen Änderungen im Beweisverfahren, die vor allem in der Einführung von neuen Beweismitteln und neuen Beweiswürdigungsgrundsätzen zum Ausdruck kamen. Das Ziel war es, dem nationalsozialistischen Anspruch auf "biologische Wahrheitsfindung" Genüge zu tun. Bei den Beweismitteln wurde teilweise an Entwicklungen angeknüpft, die schon vor 1938 eingesetzt hatten. So war das von Rassenbiologen entwickelte erbbiologisch-

\footnotetext{
${ }^{76}$ Gestapo, Mitteilung an das LG Wien (28. 8. 1941) WStLA 2.3.5.A24, Kart. 328/9/41, 9 Cg 94/41.

77 LG Wien 16. 9. 1942, 9 Cg 94/41 (unbekannt).

${ }^{78}$ Dieser Abschnitt entspricht BUKOR, Zivilrecht und Ideologie 176-185.

${ }^{79}$ Vgl. RG 29. 4. 1940, VIII 35/40; SwOBODA, ABGB 120.

${ }^{80}$ Vgl. RG 29. 9. 1943, VII 89/43.
} 
anthropologische Gutachten bereits vor dem Anschluss in vereinzelten Zivilprozessen vor österreichischen Gerichten zum Einsatz gekommen, erst in der NS-Zeit kam ihm jedoch eine zentrale Rolle zu. Darüber hinaus wurden gelegentlich auch gänzlich neuartige Beweismittel verwendet, die zumeist in engem Zusammenhang mit NS-Gedankengut standen. Dazu zählten vor allem die Gutachten von Genealogen und Sippenforschern, die sich mit der "rassischen" Abstammung einzelner Personen beschäftigten.

\section{Die Blutuntersuchung und das erbbiologisch- anthropologische Gutachten}

Ein wesentliches Element der Abstammungsverfahren stellten die Blutgruppenuntersuchung und das „erbbiologisch-anthropologische“ Gutachten dar. ${ }^{81}$ Hinsichtlich der Blutgruppenuntersuchung hatte sich zum Zeitpunkt des "Anschlusses" bereits lange die Ansicht durchgesetzt, wonach bei sachgemäßer Durchführung der volle Nachweis erbracht werden könnte, dass ein bestimmter Mann als Erzeuger des Kindes ausgeschlossen war. ${ }^{82}$ Nur vereinzelt fanden sich noch bis Ende der 1930er Jahre Stimmen, die die Zuverlässigkeit der Methode anzweifelten. Vor allem Unterstützer der morphologischen Ähnlichkeitsanalyse, wie der "Rassenbiologe" Walter Scheidt, sahen in der Blutgruppe "nicht mehr als irgendein erbliches Merkmal, und immer weniger als verschiedene erbliche Merkmale“ .83 Vor diesem Hintergrund verlangte er, dass die Blutgruppenuntersuchung nur als ein Aspekt im Rahmen der erbbiolo-

\footnotetext{
${ }^{81}$ Teilweise wurde unter erbbiologisch-anthropologischem Gutachten auch die Kombination von Blutuntersuchung und morphologischer Ähnlichkeitsanalyse verstanden.

${ }^{82}$ Vgl. RG 23. 4. 1941, VIII 22/41; LG Wien 20. 3. 1941, 15 Cg 424/40 (Wintersperger); LG Wien 22. 7. 1941, 18 Cg 120/40 (Frisch).

${ }^{83}$ Zitiert nach RÜCKER, Erbbiologische Begutachtung 177.
}

gisch-anthropologischen Untersuchung gelten sollte. Diese Ansicht blieb jedoch eine für die Judikatur unbedeutende Mindermeinung, denn mit der AV des Reichsjustizministers vom 10. August $1936^{84}$ wurde nach Einholung eines Gutachtens von staatlicher Seite bestätigt, dass das Verfahren zur Blutgruppenbestimmung bei sachgemäßer Durchführung ausreichend Sicherheit besitze, um die offenbare Unmöglichkeit der Abstammung nachzuweisen. Anordnungen, wie eine solche "sachgemäße Durchführung" auszusehen hätte, beinhaltete die AV ebenfalls.

Das Reichsgericht entschied daher, dass Blutgruppenuntersuchungen in Abstammungsverfahren von Amts wegen durchgeführt werden mussten, sofern die Möglichkeit dazu bestand. Das Unterlassen einer solchen Untersuchung begründete bei einer Vaterschaftsanfechtung, in der die Vaterschaft eines Dritten an dem Kind vorgebracht wurde, einen erheblichen Verfahrensmangel, selbst wenn die Parteien eine solche nicht beantragt hatten. ${ }^{85}$

\section{Die Pflicht zur Durchführung erb- und rassekundlicher Untersuchungen}

Auch das anthropologische Gutachten (polysymptomatischer Ähnlichkeitsvergleich von morphologisch körperlichen Merkmalen) kam schon seit den 1920er Jahren vor österreichischen Gerichten zur Anwendung. Bei diesem Gutachten wurden körperliche Merkmale bei Kind und Eltern verglichen, um dadurch die Wahrscheinlichkeit der Abstammung von einem gesetzlichen Elternteil festzustellen. Obwohl ihre Beweiskraft umstritten war, galt es auch schon vor 1938 als erheblicher Verfahrensmangel, wenn in einem Abstammungsverfahren eine von einer der Parteien beantragte Ähnlichkeitsanalyse nicht berücksichtigt wurde. Die sich

\footnotetext{
${ }^{84}$ KRUG, SCHÄFER, STOLZENBURG, Strafrechtliche Verwaltungsvorschriften ${ }^{2}$ 260-261.

${ }^{85}$ RG 19. 8. 1940, VIII 377/39.
} 
somit schon vor 1938 abzeichnende Tendenz in der Rechtsprechung, der erb- und rassekundlichen Untersuchung eine größere Beweiskraft zuzugestehen, verstärkte sich nach 1938 zunehmend. Dies lässt sich anhand der höchstrichterlichen Rechtsprechung des $\mathrm{OGH}^{86}$ und des Reichsgerichts nachweisen. Die NichtDurchführung eines von einer der Parteien beantragten erbbiologischen Gutachtens stellte in der Regel einen Verfahrensmangel dar.

Erb- und rassekundliche Erhebungen waren nur dann einzuholen, wenn ein bestimmter anderer (insbesondere "artfremder") Mann als Vater genannt wurde, da andernfalls die Vergleichsgrundlage für eine solche Untersuchung fehlte. ${ }^{87}$ Während die Hinzuziehung erbbiologischanthropologischer Untersuchungen in Fällen, in denen es um eine ,jüdische Abstammung" ging, obligatorisch war, blieb der Einsatz der Gutachten, in denen alle Beteiligten "deutschblütig“ waren, im Ermessen des Richters. Genauso blieb es im Ermessen des Richters, inwiefern er die Ergebnisse der Gutachten in seiner Entscheidung berücksichtigte, da der Beweiswert der Gutachten umstritten war. ${ }^{88}$

Die wachsende Bedeutung erfolgte jedoch nicht nur auf Basis einzelner gerichtlicher Entscheidungen, sondern wurde auch gesetzlich vorangetrieben. Durch die enge Verknüpfung des nationalsozialistischen Rassenkonzepts mit der anthropologischen und rassenbiologischen Forschung setzte der NS-Staat bei der „rassischen Einteilung“ einzelner Personen großes Vertrauen in die Gutachten der diversen rassebiologischen und anthropologischen Institute. Mit dem verstärkten Rückgriff auf „Experten“ in zivilrechtlichen Verfahren entsprach man der Forderung nach einer "völkischen Justiz", die nicht

\footnotetext{
${ }^{86}$ Vgl. OGH 2 Ob 325/38 EvBl 1938/372.

${ }^{87}$ RG 17. 11. 1943, VII 79/43; vgl. auch RG 18. 7. 1944, VII 83/44

${ }^{88}$ Vgl. PRZYREMBEL, Rassenschande 162.
}

mehr zwischen Rechts- und Lebenstatsachen unterschied. 89

Daher ordnete man durch die AV zur Beteiligung der Reichsstelle für Sippenforschung bei erb- und rassekundlichen Untersuchungen vom 10. Februar 193990 an, dass in Abstammungsverfahren erbbiologische Untersuchungen verpflichtend anzuordnen waren, wenn es um die Abstammung von einem ,jüdischen" Elternteil ging. Auch eine Unterlassung einer solchen Untersuchung mit der Begründung, dass diese angesichts der Sachlage keine Auswirkung auf die ablehnende Entscheidung hätte, wurde als Verfahrensmangel bewertet. ${ }^{91}$ Hingegen wurde ausnahmsweise von der Einholung eines Gutachtens abgesehen, wenn die Blutgruppenuntersuchung ein eindeutiges Ergebnis zu Tage gebracht hatte ${ }^{92}$ und wenn Zeugenaussagen und Meldedaten übereinstimmend ergaben, dass eine Person in der kritischen Zeit nicht im Land war. ${ }^{93}$

Die für die Erstellung der Gutachten zuständigen Institutionen waren aufgrund der großen Anzahl von Verfahren und des kriegsbedingten Personalmangels überfordert, allen Ansuchen der Gerichte nachzukommen. Zur Entlastung dieser Einrichtungen ordnete $\S 19$ der Zweiten KriegsmaßnahmenVO an, dass erb- und rassekundliche Untersuchungen nur dann einzuholen waren, wenn sie erforderlich waren, um klarzustellen, ob oder in welchem Grade jemand „,artfremden“ Blutes sei. ${ }^{94}$

\footnotetext{
${ }^{89}$ FRANCK, Juristen und Sachverständige 210.

90 Zitiert nach KRUG, SCHÄFER, STOLZENBURG, Strafrechtliche Verwaltungsvorschriften ${ }^{2}$ 773-774.

${ }^{91}$ OLG Wien 4. 6. 1940, 2 R 356/40.

92 RG 28. 5. 1941, VIII 43/41.

${ }^{93}$ Z.B. LG Wien 12. 7. 1940, 30 Cg 277/39 (Michlmayr); LG Wien 20. 10. 1942, 6 Cg 93/42 (Wintersperger); LG Wien 4. 12. 1940, 4 Cg 93/40 (Jedliczka).

${ }^{94}$ RG 28. 2. 1945, VII 12/45.
} 


\section{Die Bedeutung erb- und rassekundlicher Untersuchungen}

Die Bedeutung erbbiologischer Gutachten war in erster Linie von der konkreten Fallkonstellation abhängig: Gegen ein widersprechendes Resultat einer Blutuntersuchung konnte sich das erbbiologische Gutachten niemals durchsetzen. Daher entschied das Reichsgericht, dass die Nicht-Beantragung eines erbbiologischanthropologischen Gutachtens in solchen Fällen auch keinen Verfahrensmangel darstelle. ${ }^{95}$ Selbiges galt, wenn die Sachverhaltsfeststellung ergab, dass sich der gesetzliche Vater laut seiner Krankengeschichte in der kritischen Zeit in einer psychiatrischen Anstalt befunden hatte. ${ }^{96}$ Auch in anderen Fällen konnte das Ergebnis eines erbbiologischen Gutachtens verworfen werden, bspw. dann, wenn das Erstgericht den letzten Geschlechtsverkehr zwischen der Mutter und dem Beklagten 303 Tage vor dem Geburtstermin festgestellt hatte. In diesem Fall galt die Zeugungsmöglichkeit durch den vom Gutachten präferierten Vater als so gut wie ausgeschlossen. ${ }^{97}$ Obwohl das erbbiologische Gutachten in vielen Fällen eine wichtige Rolle spielte, zeigt dieses Beispiel, dass der anthropologischen Methode die geringste Beweiskraft unter den „naturwissenschaftlichen" Beweisen zuerkannt wurde.

Darüber hinaus war die Aussagekraft anthropologischer Gutachten in der Praxis dadurch beschränkt, dass sie in ihrem Ergebnis laut reichsgerichtlicher Rechtsprechung zumindest einen hohen Grad der Wahrscheinlichkeit der Abstammung bzw. Nicht-Abstammung attestieren mussten, um überhaupt Einfluss auf das Urteil zu haben. ${ }^{98}$ In den Gutachten wurde das Vorliegen einer „hohen Wahrscheinlichkeit" nur selten

\footnotetext{
${ }^{95}$ RG 28. 5. 1941, VIII 43/41.

${ }^{96}$ LG Wien 11. 11. 1943, 20 Cg 271/43 (Wintersperger).

${ }^{97}$ RG 8. 5. 1941, VIII 31/41.

${ }^{98}$ Vgl. RG 29.1. 1941, VIII 145/40; RG 23. 6. 1943, VII

60/43; 4 Cg 55/42 (14. 12. 1942).
}

explizit bestätigt. Allerdings reichte zumeist auch das Ergebnis, dass die Abstammung vom angeblichen Vater beträchtlich höher sei als die vom gesetzlichen Vater. Doch selbst wenn ein anthropologisches Gutachten den erforderlichen Grad der Wahrscheinlichkeit feststellte, musste das Gericht dem Ergebnis nicht folgen. Es stand dem Richter in Ermangelung einer entsprechenden fachlichen Ausbildung zwar nicht zu, das Ergebnis des Gutachtens abzulehnen, er konnte aber gemäß § 272 ZPO den Beweiswert des Gutachtens nach freiem Ermessen beurteilen. ${ }^{99}$ Vor diesem Hintergrund entschieden sich einzelne Richter dazu, dem Ergebnis nicht zu folgen, weil das erbbiologische Gutachten nur auf Laienfotographien basierte, 100 zwei widersprüchliche Gutachten vorgelegt wurden ${ }^{101}$ oder die Schlüsse, die der Sachverständige aus dem Gutachten gezogen hatte, nicht überzeugen konnten. ${ }^{102}$ LGR Wintersperger ging sogar so weit, ein Gutachten abzulehnen, in dem festgestellt wurde, dass die Vaterschaft des angeblichen Vaters sehr wahrscheinlich, die des gesetzlichen Vaters jedoch unwahrscheinlich sei. Seine Entscheidung begründete er damit, dass es sich bei dem Gutachten nur um eine Wahrscheinlichkeitsaussage

\footnotetext{
${ }^{99}$ Siehe Fehler! Verweisquelle konnte nicht gefunden werden.

100 Z.B. LG Wien 29. 12. 1942, 26 Cg 79/42 (Hiltscher). ${ }^{101}$ LG Wien 19. 2. 1944, 25 Cg 14/43 (Judex); andere Richter entschieden sich für das dem Kläger günstigere Gutachten mit der Begründung, dass dieses überzeugender sei. In einem Fall bezeichnete einer der Gutachter (Eberhard Geyer) den Bau der Nase als Merkmal, das bei „Mischlingen mitteleuropäischer und vorderasiatisch-orientalischer Rassenelemente" häufig auftrete, während der andere (Josef Wastl) darin eine "auf Mischung der nordischen mit der dinarischen Rasse beruhende Extrembildung" erblickte. Der Richter, LGR Sarauer, folgte in diesem Fall dem zweiten Gutachten (LG Wien 23.7.1940, 19 Cg 53/40 [Sarauer]). ${ }^{102}$ LG Wien 24. 10. 1942, 28 Cg 284/41 (Hiltscher).
} 
handle, die alleine eine Unmöglichkeit der Vaterschaft noch nicht beweise. ${ }^{103}$

In der bisherigen Forschung findet sich die Behauptung, dass der Ausgang von Abstammungsprozessen fast ausschließlich von den Gutachten der gerichtlich beeideten Sachverständigen abhing. ${ }^{104}$ Wirft man einen Blick auf die Gerichtsakten des LGZ/LG Wien, wird diese Aussage nur teilweise bestätigt: Noch im Jahr 1940 wurde ein beachtlicher Teil der Verfahren ohne erbbiologisches Gutachten und teilweise auch entgegen dem Ergebniss des Sachverständigen entschieden. Von 38 relevanten Urteilen ${ }^{105}$ basierten 15 allein auf Zeugenaussagen und Urkunden, in drei Urteilen wurde dem Sachverständigen nicht gefolgt. ${ }^{106}$ Das heißt, in beinahe der Hälfte aller Fälle hatte das erbbiologische Gutachten keine Bedeutung.

Wie das Ergebnis eines Gutachtens umgedeutet werden konnte, zeigt eine Entscheidungsbegründung von LGR Lahr: Während der Sachverständige Tuppa den gesetzlichen Vater für den wahrscheinlichen Erzeuger hielt und mangels Bildmaterials keine Aussage über die Abstammung vom angeblichen Vater traf, bezog sich LGR Lahr im Urteil nur auf die Feststellung, dass die beiden Kläger „keine typischen Merkmale für das jüdische Volk" aufwiesen. Des Weiteren führte er aus:

${ }^{103}$ LG Wien 28. 5. 1942, 28 Cg 147/41 (Wintersperger); Die beklagte Partei wurde in diesem Fall weniger als 3 Wochen nach diesem Urteil deportiert und in weiterer Folge umgebracht (DÖW-Datenbank,

www.doew.at/personensuche [1. 7. 2017]).

${ }^{104}$ FUCHS, Rasse 311; vgl. auch: FRANCK, Juristen und Sachverständige 209.

${ }^{105}$ Hier sind nur Urteile berücksichtigt, in denen die Entscheidung auf einem erbbiologischen Gutachten basieren könnte. Formelle Abweisungen und Urteile, die schon aufgrund einer Blutgruppenuntersuchung entschieden wurden, bleiben unberücksichtigt.

${ }^{106}$ Zugunsten des Klägers: LG Wien 23. 7. 1940,

19 Cg 53/40 (Sarauer); LG Wien 14. 9. 1940, 8 Cg 30/40

(Lahr); zuungunsten des Klägers: LG Wien 5. 12. 1942,

25 Cg 289/40 (Hiltscher).
„Wenn sie [die Kläger] sich aus dem jüdischkaufmännischen Milieu heraus, in dem sie aufwuchsen, zum Offiziersberuf hingezogen fühlten, gegen den Willen ihrer Eltern zum Katholizismus übertraten, während eines grossen Teiles des Weltkrieges als Reserveoffiziere an den verschiedensten Fronten kämpften und mehrfach militärische Auszeichnungen erlangten, so kann dies nur so erklärt werden, dass in ihnen neben der ja auf jeden Fall von ihrer Mutter her bestehenden jüdischen auch eine arische Erbmasse vorhanden sein muss, und deuten obige Details darauf hin, dass diese von dem erwähnten ungarischen Offizier stammt. ${ }^{\prime 107}$

Ein anderes Bild zeichnet sich jedoch im Jahr 1942 ab. Von 71 relevanten Urteilen findet sich ein einziges Urteil ${ }^{108}$ in dem ein erbbiologisches Gutachten hätte durchgeführt werden können, aber auf eine Durchführung verzichtet wurde. In drei Fällen entschieden die Richter entgegen dem eindeutigen Ergebnis des erbbiologischen Gutachtens zuungunsten des Klägers.

Vor dem Hintergrund, dass sich zwischen 1940 und 1942 die richterliche Zuteilung stark wandelte und in Fällen, in denen es um die Anfechtung der "jüdischen“ Abstammung ging, ab 1941 nur mehr ein kleiner Kreis an Richtern entschied, ist die veränderte Bedeutung des erbbiologischen Gutachtens auch in Zusammenhang mit den personellen Veränderungen zu sehen. Jene Richter, die 1940 teilweise auf die Einholung eines erbbiologischen Gutachtens verzichtet ${ }^{109}$ oder sogar entgegen dem gutachtlichen Ergebnis zugunsten der Klagspartei entschieden hatten, ${ }^{110}$ waren ab 1942 nur mehr vereinzelt in solchen Verfahren zuständig. Jene Richter, die 1941 und 1942 bei Verfahren zur Anfechtung der ,jüdischen“ Abstammung auf-

\footnotetext{
${ }^{107}$ LG Wien 14. 9. 1940, 8 Cg 30/40 (Lahr).

${ }^{108}$ LG Wien 15. 10. 1943, 25 Cg 51/42 (Judex).

${ }^{109}$ Bernard, Fryda, Gabler, Hotzy, Jedliczka Kuch, Lahr, Midlarz, Sarauer, Stahl, Straniak, Weisswasser.

${ }^{110}$ Lahr, Sarauer.
} 
scheinen, waren 1940 entweder den erbbiologischen Gutachten gefolgt oder hatten sogar entgegen dem Gutachten das Klagebegehren abgewiesen. ${ }^{111}$ Davon abgesehen ist bei den Urteilen, in denen auf ein Gutachten verzichtet wurde, auch zu berücksichtigen, ob sich in dem konkreten Fall der Sachverhalt aufgrund der gesamten Beweislage so darstellte, dass ohne Gutachten entscheiden werden konnte.

\section{Zusammenfassung/Conclusio}

Durch die Untersuchung der abstammungsrechtlichen Gerichtsakten konnten neue Erkenntnisse über die Veränderungen des österreichischen Abstammungsrecht in der NS-Zeit gewonnen werden, aber auch über die Mechanismen, Faktoren und Akteure, die die Modifzierung und Ideologisierung der Rechts bewirkten.

Eine zentrale Rolle spielte freilich der nationalsozialistische Gesetzgeber. So lässt sich die Aufgabe fundamentaler zivilrechtlicher Prinzipien im legislativen Wege nachweisen. Die Ablöse des Dispositionsgrundsatzes durch das Prinzip der materiellen Wahrheit (Untersuchungsgrundsatz) äußerte sich nicht zuletzt darin, dass dem Staatsanwalt erweiterte Kompetenzen im Bereich des Zivilrechts zukamen. Die gesetzliche Grundlage des österreichischen Abstammungsrechts wurde jedoch relativ spät, in den Jahren 1943 und 1944 geändert.

Doch schon für die Zeit davor lassen sich auch andere Faktoren feststellen, die das österreichische Abstammungsrecht beeinflussten und oft auch ideologische Spuren in der Judikatur hinterließen. Dazu zählen z.B. die Rechtsprechung des Reichsgerichts zum BGB, die zunehmende Einmischung von Behörden und Parteiorganisationen und nicht zuletzt die "Biologisierung" des Diskurses über Familien- und Abstam-

111 Z.B. LG Wien 5. 12. 1942, 25 Cg 289/40 (Hiltscher). mungsrecht. Die nicht unwesentlich auf dem Kindeswohl und der ehelichen Familie basierenden Abstammungsregelungen des ABGB wurden - vor allem dort, wo es aus „rassepolitischen und erbgesundheitlichen“ Gründen als notwendig erachtet wurde - durch einen Primat der „materiellen Wahrheit" ersetzt, der seine Berechtigung auf die fragwürdigen Forschungen der „Erb- und Rassenbiologie“ stützte. So wurde es zuerst Usus und später Pflicht, in Fällen, in denen die ,jüdische Abstammung“ des Klägers zur Disposition stand, ein erb- und rassebiologisches Gutachten einzuholen. Interessanterweise zeigt gerade der richterliche Umgang mit den Gutachten die Grenzen der „Verwissenschaftlichung" auf. Da diese Gutachten kaum die Sicherheit boten, die verlangt wurde, spielten sie selten eine entscheidende Rolle.

Ein weiteres Ergebnis der Untersuchung betrifft die Auswirkung der "Rassepolitik“ auf das Zivilrecht, die sich zahlenmäßig nachweisen und darstellen ließs. So ist der starke Anstieg der Ehelichkeitsanfechtungen nach 1938 zu einem Großteil auf Anfechtungen zurückführen, die gegen die Abstammung von einem ,jüdischen Elternteil“" gerichtet waren. Diese machten im Jahr 1941 sogar fast die Hälfte aller Ehelichkeitsanfechtungen aus.

Ein letzter Aspekt der Untersuchung betrifft die Rückschlüsse auf die Einstellung und Ausrichtung einzelner Richterpersönlichkeiten und auf die NS-Personalpolitik im Gerichtswesen. In diesem Zusammenhang sind zwei Umstände bemerkenswert. Zum einen die auffällig hohe Anzahl an Klagsabweisungen durch einzelne Richter in Verfahren, in denen der Kläger die Abstammung von seinem ,jüdischen Elternteil“ anzufechten versuchte. Und zum anderen der Umstand, dass gerade diese Richter in weiterer Folge überproportional in solchen Verfahren anzufinden sind. Somit kann der Schluss gezogen werden, dass bestimmte Richtertypen, die für eine besonders restriktive Vorgangsweise standen, bevorzugt herangezogen wurden. 


\section{Korrespondenz:}

Mag. Dr. Benjamin BUKOR

benjamin.bukor@gmail.com

ORCID-Nr. 0000-0002-5265-348X

\section{Abkürzungen:}

$\begin{array}{ll}\text { AV } & \text { Allgemeinverfügung } \\ \text { DÖW } & \begin{array}{l}\text { Dokumentationsarchiv des } \\ \text { österreichischen Widerstandes }\end{array} \\ \text { DJ } & \text { Deutsche Justiz } \\ \text { DR } & \text { Deutsches Recht } \\ \text { DR (WrA) } & \text { Deutsches Recht (Wiener Ausgabe) } \\ \text { EvBl } & \text { Evidenzblatt der } \\ & \text { Rechtsmittelentscheidungen } \\ \text { FamRechtsVO } & \text { Verordnung über die Angleichung } \\ & \text { familienrechtlicher Vorschriften } \\ & \text { vom 6. Februar 1943 } \\ \text { LGDir } & \text { Landesgerichtsdirektor } \\ \text { LGR } & \text { Landesgerichtsrat } \\ \text { LGZ } & \text { Landgericht/Landesgericht } \\ & \text { für Zivilrechtssachen } \\ \text { RdErl } & \text { Runderlass } \\ \text { RMI } & \text { Reichsministerium des Innern } \\ \text { WStLA } & \text { Wiener Stadt- und Landesarchiv } \\ \text { ZStfjA } & \text { Zentralstelle für jüdische } \\ & \text { Auswanderung }\end{array}$

Siehe auch das allgemeine Abkürzungsverzeichnis: [http://www.rechtsgeschichte.at/files/abk.pdf]

\section{Literatur:}

Gabriele ANDERL, Dirk RupNOW, Alexandra-Eileen WENCK, Die Zentralstelle für Jüdische Auswanderung als Beraubungsinstitution, Veröffentlichungen der Österreichischen Historikerkommission. Vermögensentzug während der NS-Zeit sowie Rückstellungen und Entschädigungen seit 1945 in Österreich Nationalsozialistische Institutionen des Vermögensentzuges (Wien 2004).

Benjamin BUKOR, Zivilrecht und Ideologie. Die Entwicklung des österreichischen Abstammungs- rechts in der NS-Zeit unter Berücksichtigung der Judikatur des LGZ bzw. LG Wien und des Reichsgerichts (iur. Diss., Univ. Wien 2015).

Heinz Christian DAUT, Untersuchungen über den Einfluß nationalsozialistischer Anschauungen auf die Rechtsprechung des Reichsgerichts in Zivilsachen bei der Anwendung einiger nationalsozialistischer Gesetze (iur. Diss., Univ. Göttingen 1965).

Brigitte FucHS, „Rasse“, „Volk“, Geschlecht. Anthropologische Diskurse in Österreich 1850-1960 (Frankfurt am Main 2003).

Lorenzen FRANCK, Juristen und Sachverständige. Der Diskurs um die Ausgestaltung des Verfahrens mit Sachverständigen während der Zeit des Deutschen Reiches (Baden-Baden 2013).

Karl KRUG, Karl ScHÄFER, Friedrich Wilhelm STOLZENBURG (Hgg.), Strafrechtliche Verwaltungsvorschriften. Eine Sammlung der vom Reichsminister der Justiz erlassenen Verwaltungsvorschriften und wichtigen Einzelerlasse auf dem Gebiete des Strafrechts und des Strafverfahrens (Berlin 21939).

Thomas MAYER, Familie, Rasse und Genetik. Deutschnationale Eugeniken im Österreich der Zwischenkriegszeit, in: Gerhard BAADER, Veronika HOFER, Thomas MAYER (Hgg.), Eugenik in Österreich (Wien 2007) 162-183.

Wolfgang Neugebauer, Richter in der NS-Zeit. Politische und gesellschaftliche Rahmenbedingungen, in: Erika WEINZIERL, Oliver RATHKOLB, Siegfried MatTL, Rudolf G. ARdelt (Hgg.), Richter und Gesellschaftspolitik. Symposion Justiz und Zeitgeschichte 12. und 13. Oktober 1995 in Wien (Innsbruck 1997) 56-68.

Alexandra PRZYREMBEL, Rassenschande. Reinheitsmythos und Vernichtungslegitimation im Nationalsozialismus (=Veröffentlichungen des MaxPlanck-Instituts für Geschichte 190, Göttingen 2003).

RÜCKER, Erbbiologische Begutachtung bei umstrittener Vaterschaft vom Standpunkt der Juristen, in: Deutsche Zeitschrift für die gesamte gerichtliche Medizin (1937) 176-185.

Ernst Swoboda (Hg.), Das österreichische Allgemeine bürgerliche Gesetzbuch (Wien ${ }^{2} 1944$ ).

Ernst SWOBODA, Die neue Familienrechtsverordnung, in: DR (WrA) (1943) 49. 